Document downloaded from:

http://hdl.handle.net/10251/120699

This paper must be cited as:

Carrión García, A.; Genovés, V.; Pérez, G.; Paya Bernabeu, JJ.; Gosálbez Castillo, J. (2018). Flipped Accumulative Non-Linear Single Impact Resonance Acoustic Spectroscopy (FANSIRAS): A novel feature extraction algorithm for global damage assessment. Journal of Sound and Vibration. 432:454-469. https://doi.org/10.1016/j.jsv.2018.06.031

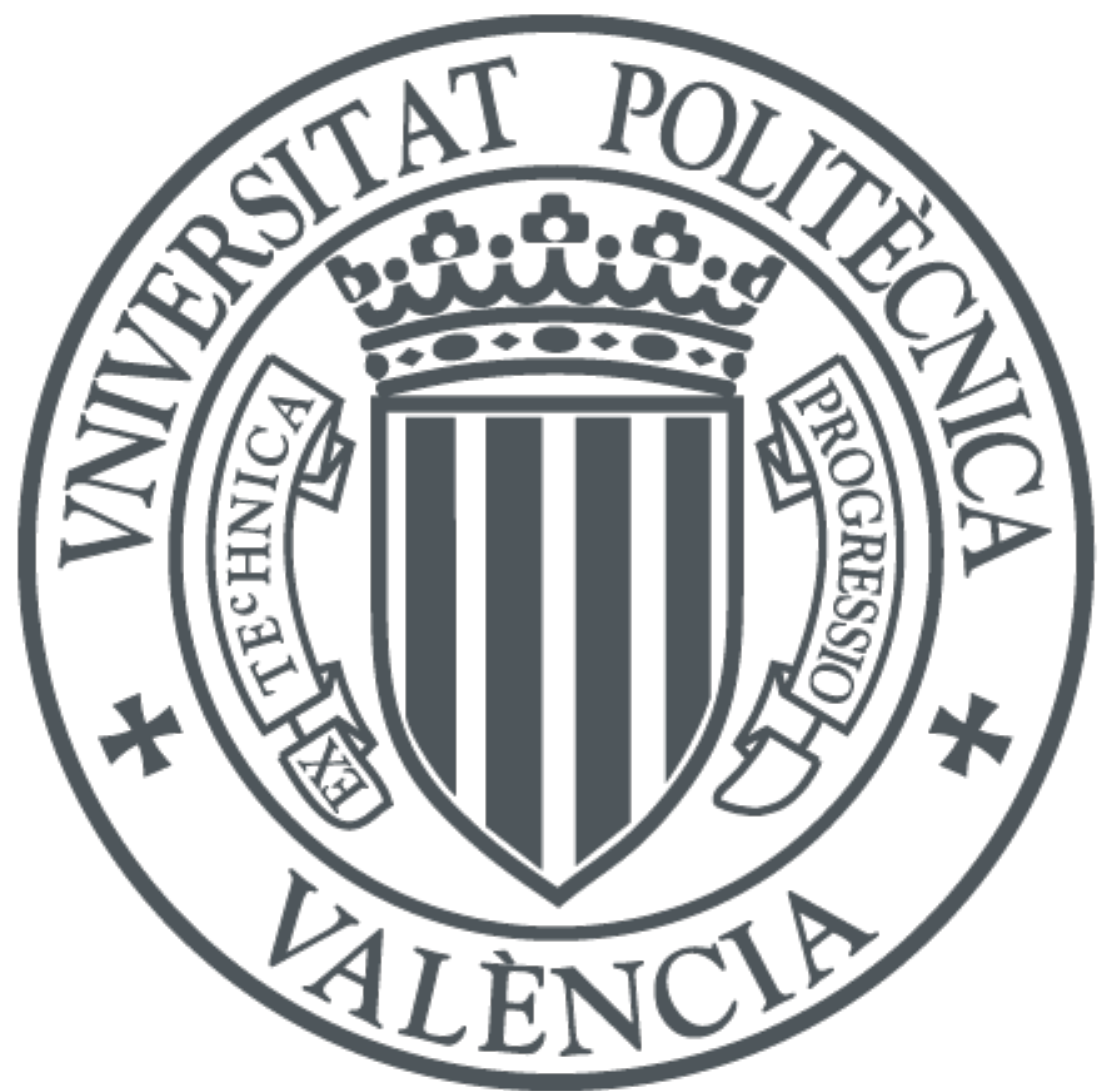

The final publication is available at

http://doi.org/10.1016/j.jsv.2018.06.031

Copyright Elsevier

Additional Information 


\title{
Flipped Accumulative Non-Linear Single Impact Resonance Acoustic Spectroscopy (FANSIRAS): A novel feature extraction algorithm for global damage assessment
}

\author{
A. Carrión ${ }^{\mathrm{a}, *}$, V. Genovés ${ }^{\mathrm{b}}$, G. Pérez ${ }^{\mathrm{a}}, \mathrm{J}$ Payá $^{\mathrm{b}}, \mathrm{J}$ Gosálbez $^{\mathrm{a}}$
}

${ }^{a}$ ITEAM, Universitat Politècnica de València, Camino de Vera, s/n 46022 Valencia, Spain

${ }^{b}$ ICITECH, Universitat Politècnica de València, Camino de Vera, s/n 46022 Valencia, Spain

\begin{abstract}
High amplitude non-linear acoustic methods have shown great potential for the identification of micro-damage in inhomogeneous materials such as concrete. Usually, these methods evaluate non-linearity parameters related to the hysteretic behaviour from the dependence of the shifts in both frequency and damping on the amplitude of the strain. A deep understanding of the reverberation phenomena has been obtained in order to introduce a novel signal processing approach called FANSIRAS (Flipped Accumulative Non-linear Single Impact Resonance Acoustic Spectroscopy). Traditional acoustic spectroscopy techniques, NIRAS (Non-linear Impact Resonance Acoustic Spectroscopy) and NSIRAS (Non-linear Single Impact Acoustic Spectroscopy), have been analysed and compared with the brand new approach when providing quantitative information related to the degree of micro-cracking in thermal damaged concrete based materials. The new resonance-based algorithm demonstrates that the non-linear non-classical parameters can be determined through a single resonance frequency measurement, obtaining the expected sensitivity to internal damage. Its simplicity and robustness may be important in industrial applications. Keywords:

20 Non-Linear Acoustic Spectroscopy, Hysteresis, NIRAS, FANSIRAS, NSIRAS, Concrete, Thermal damage
\end{abstract}

\section{Introduction}

The study of the vibration of engineered materials is obtaining high levels of interest from scientific communities due to the accuracy and facility of damage assessment $[1,2]$. Concrete is an inhomogeneous material with a mesoscopic structure, multi-scaled, from nano- to millimetric sizes, where Hertzian contact between particles predominates. In this material, there appears an anomalous resonance frequency shift (a change in the elastic constants) when it is excited by an external source. This vibration is attenuated and finally stops, returning to its primary state, as the driving amplitude decreases and low strain values are reached [3]. This dynamic phenomenon is closely related to the hysteretic and non-linear behaviour of mesoscopic materials, whose deformations due to an external stress cannot be described by Hooke's law, making it imperative to add more complex

\footnotetext{
*Corresponding author E-mail adress: alcarga4@upv.es
} 
terms to the traditional elastic equations. The first studies in mesoscopic materials focusing on its dynamic behaviour were carried out for rocks and granular solids like sandstone and limestone, reporting non-linear and hysterectic responses, while trying to model this phenomenon with complex equations [4, 5]. Van Den Abeele et al. [6] presented a one-dimensional constitutive relation between the stress and strain in this new class of materials (Eq. 1):

$$
K(\epsilon, \dot{\epsilon})=K_{0} \cdot\left[1-\beta \epsilon-\delta \epsilon^{2}-\alpha[\Delta \epsilon+\epsilon \cdot \operatorname{sign}(\dot{\epsilon})]\right]
$$

where $K$ is the non-linear hysteretic modulus with $K_{0}$ denoting the linear modulus, $\beta$ and $\delta$ the classical quadratic and cubic non-linear parameters, respectively, $\epsilon$ the strain, $\Delta \epsilon$ the strain amplitude in a cycle, $\dot{\epsilon}$ the strain rate, and the parameter $\alpha$ is a measure proportional to the hysteresis of the material. Due to the proportionality between the resonance frequency of an element and its elastic constants, this equation describes the principal resonance frequency mode at a given strain amplitude.

Because of its granular and inhomogeneous structure, concrete can be considered a synthetic rock, with static and dynamic behaviour similar to other materials studied in geophysics and soil engineering. This material is the most important component in building and civil structures, its safety and control over its life time being critical. The continuous development and enhancement of new non-destructive techniques that allow controlling the quality and health of the concrete has become essential. Recently, several studies have focused on monitoring damage to concrete using non-linear elastic wave spectroscopy (NEWS) methods, a powerful new tool that takes advantage of the properties of mesoscopic materials in that they are more sensitive to cracks, flaws, and distributed defects. Due to the non-linearity of the material, a wave can become distorted, generating new frequency components (subharmonics and higher harmonics) during the propagation of a monochromatic wave [7], mixing waves of different frequencies [6]. Also, under resonance conditions, changes in the resonance so frequency occur as a function of the driving amplitude [8]. These phenomena are very weak in undamaged materials, but remarkably larger in damaged materials [9].

In the last decade, several authors have successfully characterized the damage of materials by means of NEWS methods [10]. These experiments are based on the same phenomenological model and they can be categorized by the source of excitation (acoustic, ultrasonic or impact) and the number of signals injected into the system (one or several). The apparatus used in the different studies varies substantially, but the essence of the determination of the non-linear properties remains the same in all cases. Some authors have used an acoustical source (electroacoustic transducer, mainly) in order to induce the element under examination into a steady state of reverberation. Some investigations have evaluated this behaviour by using several signals, involving the use of frequency sweeps around the principal mode of vibration of the element, at different input amplitudes (SIngle MOde Nonlinear Resonance Acoustic Spectroscopy, SIMONRAS [4, 8]; Nonlinear Wave Modulation Spectroscopy, NWMS [6]), and others have used one single signal and computed the attenuation of the material when the source 
of motion stops (Nonlinear Resonance Spectroscopy, NRS[11]). The signals were recorded by accelerometers or laser vibrometers. In ultrasonic excitation, there has been used a piezoelectric transducer for signal transmission and another transducer or laser vibrometer as the receiver. In this case, frequency sweeps at different input voltages have been employed to induce the element into a steady state of vibration (SIngle MOde Nonlinear Resonance Ultrasonic Spectroscopy, SIMONRUS [6]; Nonlinear Resonance Ultrasonic Spectroscopy, NRUS [1215]). New research is focused in impact spectroscopy because of its low cost and the easy excitation of the elements under study. An impact hammer has been used to induce the vibration of the element and record the acceleration experienced by the specimen, by means of an accelerometer attached to its surface (Nonlinear Impact Resonance Acoustic Spectroscopy, NIRAS [16-18]; Nonlinear Single Impact Resonance Acoustic Spectroscopy, NSIRAS [19]; and Impact Nonlinear Reverberation Spectroscopy, INRS [20]).

A summary of NEWS methods is shown in Table 1.

Table 1: Summary of non-linear elastic wave spectroscopy methods according to the type of excitation source and the number of signals.

\begin{tabular}{llll}
\hline & Excitation source & & Impact \\
\cline { 2 - 3 } Number of signals & Acoustic wave & Ultrasonic wave & NIRAS [16-18] \\
\hline 1 & SIMONRAS [4, 8], NWMS [6] & NWMS [6], SIMONRUS [3], NRUS [12-15] & NSIRAS [19], INRS [20] \\
\hline
\end{tabular}

As can be observed in the literature, the recent scientific research in NEWS techniques is focused on impact spectroscopy with one single signal, because of the obvious advantage in the testing time and probe conditioning. In the present study, a brand-new technique is developed, following the original methodology seen in NIRAS and inspired by a different signal processing procedure proposed by Eiras et al.[19] and Dahlen et al. [20]. The aim of this paper is to obtain a valid technique completely equivalent to NIRAS but developed with one single impact, by means of the analysis of the signal processing procedure. This technique has been tested on the thermal damage assessment of specimens of Portland cement mortar. This paper is organized as follows: In Section 1, the state of the art of NEWS techniques and the scope of the present paper are described. The mathematical background and signal processing issues of the different techniques are described in Section 2. The materials, specimens, and test layout used to obtain the results of the present study are described in Section 3. The results obtained from the new technique and a comparison of the data obtained from the different impact resonance acoustic spectroscopic methods are found in Section 4. In Section 5 the conclusions of this study are presented.

\section{Mathematical Background}

Most of the usual threats to the durability of concrete result in distributed micro-cracking damage, which favours a hysteretic behaviour in the stress-strain relation. In typical resonance frequency tests, this hysteretic behaviour manifests itself as a downward shift of the resonance frequency with increasing amplitude of the excitation. Along with the frequency-amplitude dependence, the damping properties also become dependent 
on the amplitude. The origin of this behaviour appears to be in the rubbing between the interfaces of internal defects produced upon mechanical wave propagation. Besides these effects, a considerable effort has to be made to understand the underlying physical phenomena to develop new nondestructive non-linear resonance spectroscopy based techniques.

A discrete reverberation signal, $y[n]$, may be studied as the product of an exponentially decaying function, $a[n]$, and an exponential time-varying phase signal, $s[n][11,21]$. The instantaneous frequency exponentially varies from a minimum frequency $f_{\min }\left(f[0]=f_{\min }\right)$ up to a maximum frequency $f_{\max }$ taken at the end of the sampling interval $\left(f[N-1]=f_{\max }\right)$, resulting:

$$
f[n]=f_{\min }+\Delta f \cdot\left(1-\mathrm{e}^{-\gamma n T_{s}}\right), n \in\{0, \ldots, N-1\}
$$

where $\Delta f=\frac{f_{\max }-f_{\min }}{1-\mathrm{e}^{-} \gamma(N-1) T_{s}}$ controls the exponential frequency swept. Therefore, a reverberation signal $y[n]$ may be modelled as:

$$
y[n]= \begin{cases}a[n] \cdot s[n]=\overbrace{\left(a \cdot \mathrm{e}^{-\gamma n T_{s}}\right)}^{a[n]} \cdot \overbrace{\left(\sin \left(2 \pi f_{\max } n T_{s}-2 \pi \frac{\Delta f}{\gamma}\left(1-\mathrm{e}^{-\gamma n T_{s}}\right)\right)\right)}^{s[n]}, & n \in\{0, \ldots, N-1\} \\ 0, & \text { otherwise }\end{cases}
$$

where $a$ denotes the amplitude and $\gamma$ the attenuation, $T_{s}$ is the sampling period (which is the inverse of the sampling frequency, i.e. $f_{s}=1 / T_{s}$ ), and $N$ is the number of samples acquired in the sampling interval $N \cdot T_{s}$. The resulting signal is an exponentially decaying sine function with a time-varying phase, which simulates the upward resonance frequency shift observed during the analysis of resonance frequency tests. The shifts in both the resonance frequency and in the attenuation properties due to non-linear effects are then studied in the frequency domain by applying the discrete-time, discrete-frequency Fourier Transform (DFT) with $N_{\mathrm{DFT}}$ equally spaced points:

$$
Y[f]=T_{s} \cdot \operatorname{DFT}\{y[n]\}=T_{s} \cdot \sum_{n=0}^{N_{\mathrm{DFF}}-1} y[n] \cdot \mathrm{e}^{-\mathrm{j} 2 \pi f n T_{s}}, f \in\left[0,1 \cdot f_{s} / N_{\mathrm{DFT}}, \ldots,\left(N_{\mathrm{DFT}} / 2-1\right) \cdot f_{s} / N_{\mathrm{DFT}}\right] \simeq\left[0, f_{s} / 2\right]
$$

Note that the DFT has been expressed in terms of the analog frequency $(f)$ to ease the understanding of the variables involved in the technique, regardless of the digital acquisition process. Two corrections have been made to the expression: the discrete frequency samples have been expressed in terms of the analogue frequency, and the amplitude spectrum has been weighted by $T_{s}$. The number of points over which the Fourier transform is computed, $N_{\mathrm{DFT}}$, is always larger than the number of time samples, $N$, to improve the frequency visualization (zero padding). In order to avoid discrepancies between different sampling frequencies $f_{s}$, the graphical resolution in the frequency domain $\left(f_{s} / N_{\mathrm{DFT}}\right)$ is kept equal to 0.25 . 

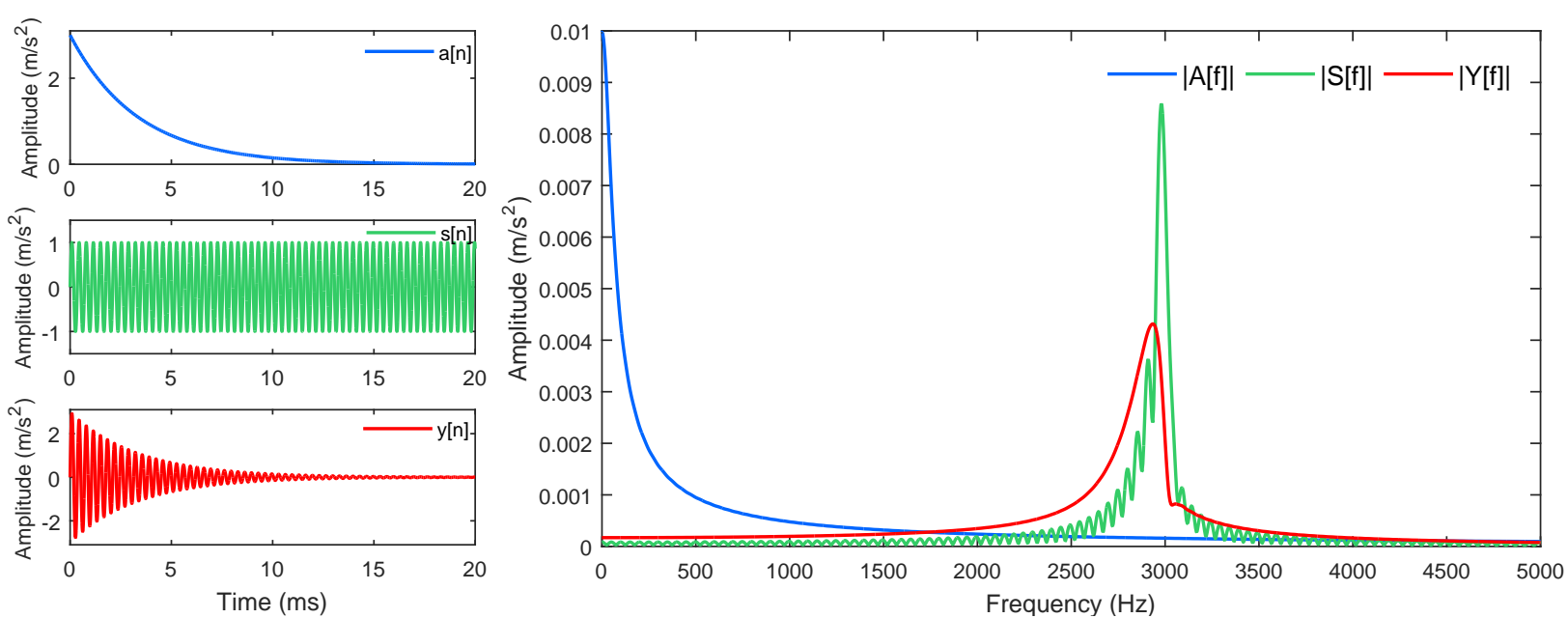

Figure 1: The left part of the panel represents the temporal aspect of the three involved signals, and the right part represents the three amplitude spectra. The blue lines are related to the exponential decay signal with $\gamma=300$ and $a=3$. The green line represents the time-varying phase sinusoidal function with $f_{\text {min }}=2.8$ $\mathrm{kHz}, \Delta f=200 \mathrm{~Hz}, f_{\max }=3 \mathrm{kHz}, f_{s}=500 \mathrm{kHz}, N=10000$. The red line represents the resulting reverberation signal.

Due to the characteristic damped non-linear reverberation signal, wherein both the frequency and the damping change as a function of time and amplitude, both these factors will influence the width and the amplitude of the resulting resonance spectral peak. Mathematically, the influence of these factors is explained by substituting Eq. 3 in Eq. 4 and applying the convolution theorem. This results in the following equation.

$$
\begin{aligned}
Y[f] & =T_{s} \cdot \sum_{n=0}^{N_{\mathrm{DFT}}-1}\left(a \cdot \mathrm{e}^{-\gamma T_{s} n} \cdot \sin \left(2 \pi f_{\max } n T_{s}-2 \pi \frac{\Delta f}{\gamma}\left(1-\mathrm{e}^{-\gamma n T_{s}}\right)\right)\right) \cdot \mathrm{e}^{-\mathrm{j} 2 \pi f n T_{s}} \\
& =T_{s} \cdot\left(\operatorname{DFT}\left\{a \cdot \mathrm{e}^{-\gamma n T_{s}}\right\} * \operatorname{DFT}\left\{\sin \left(2 \pi f_{\max } n T_{s}-2 \pi \frac{\Delta f}{\gamma}\left(1-\mathrm{e}^{-\gamma n T_{s}}\right)\right) \cdot \mathrm{e}^{-\gamma n T_{s}}\right\}\right) \\
& =T_{s} \cdot(A[f] * S[f]), \quad f \in\left[0, f_{s} / 2\right]
\end{aligned}
$$

Here, $A[f]$ is the Fourier transform of the exponentially decaying signal, $a[n]$, and $S[f]$ is the Fourier transform of the time-varying phase sinusoidal function, $s[n]$. The Fourier transform of the resulting signal, $Y[f]$, can be seen as a weighted average of the frequency and amplitude content of both of the previous signals, resulting from the convolution.

An example is shown in Figure 1 with the following parameters: $a=3, \gamma=300, f_{\min }=2.8 \mathrm{kHz}, \Delta f=200 \mathrm{~Hz}$, $f_{\max }=3 \mathrm{kHz}, f_{s}=500 \mathrm{kHz}, N=10000$. The left part of the panel represents the temporal aspect of the three involved signals, and the right part represents the three amplitude spectra. The product of any two time signals is equivalent to a convolution in the frequency domain, where the maximum amplitude of the reverberation signal, $Y[f]$, corresponds to the minimum frequency of the chirp signal involved $(2.8 \mathrm{kHz})$, and the peak width of the reverberation signal, $Y[f]$, is proportional not only to the bandwidth of the spectrum of the decreasing exponential signal (proportional to the attenuation $\gamma$ ) but also to the frequency width of the chirp signal.

Traditional non-linear resonance tests are based on the investigation of the damping variations and the shift of 


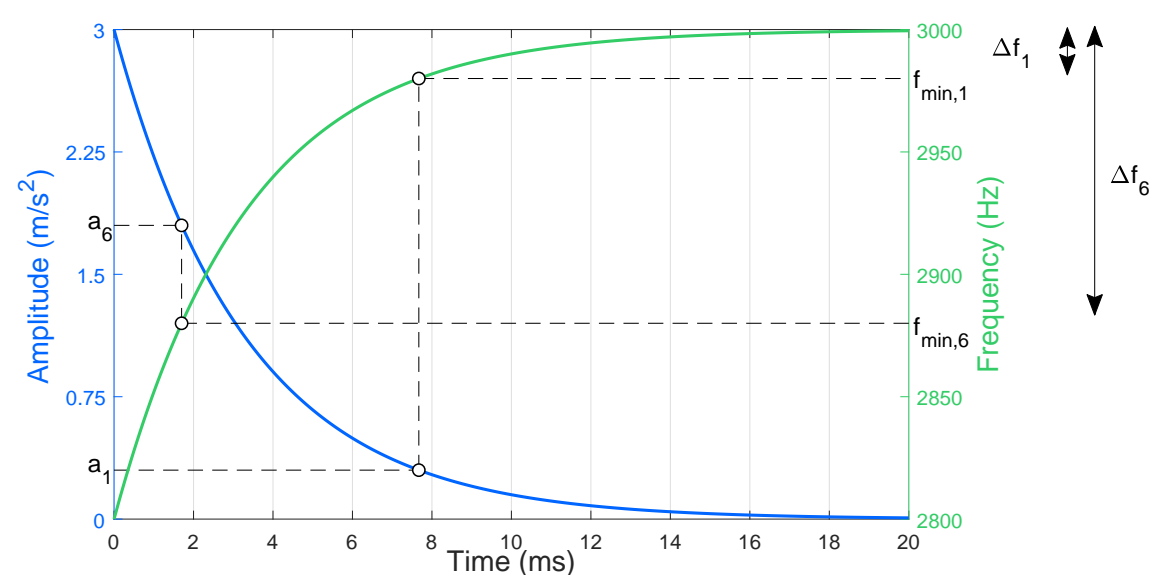

Figure 2: Temporal evolution of the instantaneous amplitude, $a[n]$ (blue line), and the instantaneous frequency, $f[n]$ (green line), according to Eq. 6 and Eq. 2 , respectively. The parameters are with $a=3, \gamma=300, f_{\min }=2.8 \mathrm{kHz}, \Delta f=200 \mathrm{~Hz}, f_{\max }=3 \mathrm{kHz}, f_{s}=500 \mathrm{kHz}$ and $N=10000$.

the resonance frequency by excitations of increasing amplitude. These effects can also be studied as results of increasing the frequency range of the underlying time-varying phase signal: a widening of the chirp spectrum as the impact energy increases. The different reverberation signals of a resonance test are denoted by $y_{i}(n)$, with $i$ the impact number and proportional to the strength (in this study, $i \in[1,10]$ ). According to Eq. 3 , both the instantaneous frequency, $f[n]$ (Eq. 2), and the amplitude envelope of a reverberation signal, $a[n]$ (Eq. 6) vary according to the attenuation $\gamma$.

$$
a[n]=a \cdot \mathrm{e}^{-\gamma n T_{s}}, n \in\{0, \ldots, N-1\}
$$

The attenuation $\gamma$ not only controls the system instantaneous amplitude but also the frequency ranging from $f_{\min }$ to $f_{\max }$ (see Figure 2). In the practice for different levels of impact, it has been checked that the attenuation $\gamma$ does not change, it only varies the maximum amplitude $a$; and its corresponding minimum frequency $f_{\min }$ following Figure 2. So that, the reverberation signals acquired in a traditional resonance test at different impact levels, $i$, may be mathematically modelled as follows:

$$
\begin{aligned}
y_{i}[n] & =a_{i} \cdot \mathrm{e}^{-\gamma n T_{s}} \cdot \sin \left(2 \pi f_{\max } n T_{s}-2 \pi \frac{\Delta f_{i}}{\gamma}\left(1-\mathrm{e}^{-\gamma n T_{s}}\right)\right), n \in\{0, \ldots, N-1\} \\
a_{i} & =i \cdot a \\
\Delta f_{i} & =\frac{f_{\max }-f_{\min , i}}{1-\mathrm{e}^{-\gamma(N-1) T_{s}}}
\end{aligned}
$$

where $a$ is the lowest impact amplitude, and $f_{\min , i}$ is the corresponding minimum frequency according to the attenuation $\gamma$, the amplitude $a_{i}$ and the relations marked in Figure 2. The reference amplitude and frequency functions correspond to the aforemention simulated experimental described in Figure 1, which corresponds to the strongest impact in a simulated resonance test. From these strongest impact curves, it is possible to determine 

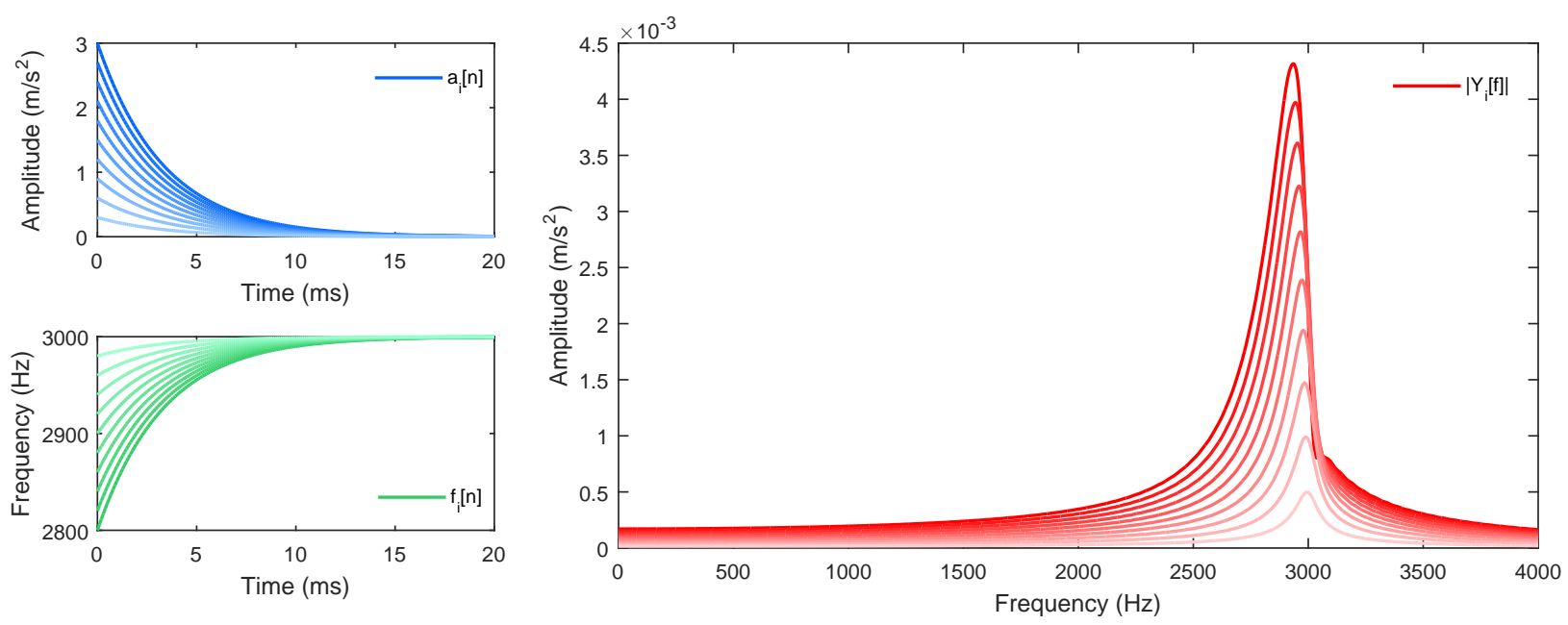

Figure 3: The left part of the panel represents the temporal evolution of the envelope amplitude $a_{i}[n]$ (blue lines) and its corresponding instantaneous frequency $f_{i}[n]$ with $i \in[1,10]$ (green lines). In the right part of the panel, the different amplitude Fourier transform of the resulting simulated signals, $Y_{i}[f]$, are plotted in red. The color legend varies from light to dark colors as the level of impact increases. The parameters of the model are $a=3, \gamma=300, f_{\text {min }}=2.8 \mathrm{kHz}$, $\Delta f=200 \mathrm{~Hz}, f_{\max }=3 \mathrm{kHz}, f_{s}=500 \mathrm{kHz}, N=10000$.

the frequency variation for lower impacts: the corresponding first impact would have an amplitude $a_{1}$ equals to 0.3 and a $f_{\min , 1}$ equals to $2980 \mathrm{~Hz}$. The corresponding sixth impact would have an amplitude $a_{6}$ equals to 1.8 and a initial minimum frequency $f_{\min , 6}$ equals to $2880 \mathrm{~Hz}$. Figure 3 presents the temporal evolution of the envelope amplitude $a_{i}[n]$ with $i \in[1,10]$ and its corresponding instantaneous frequency $f_{i}[n]$. In the right part of the panel, the different amplitude Fourier transform of the resulting simulated signals, $Y_{i}[f]$, are plotted. The color legend varies from light to dark colors as the level of impact increases. This model will be used in Section 2.4 to evaluate the different signal processing algorithms.

\subsection{NIRAS}

The technique of Non-linear Impact Resonance Acoustic Spectroscopy (NIRAS) relies on an impulse excitation of the specimen repeated with different impact strengths. The hysteresis parameter related to the resonance frequency shift $\alpha_{f}^{\text {NIRAS }}$ is obtained from a linear fit of the obtained peak amplitude spectrum $A_{i}$ (Eq. 10) versus the peak normalized frequency $\tilde{f}_{i}$ (Eq. 11) of one mode from the multiple impacts at different strengths (Eq. 12).

$$
\begin{aligned}
A_{i} & =\max \left\{\left|Y_{i}[f]\right|\right\} \\
\tilde{f}_{i} & =\max _{f}\left\{\left|Y_{i}[f]\right|\right\} \\
\frac{\tilde{f}_{0}-\tilde{f}_{i}}{\tilde{f}_{0}} & =\alpha_{f}^{\mathrm{NIRAS}} \cdot A_{i}
\end{aligned}
$$

Here, $\tilde{f}_{0}$ is the resonance frequency obtained in the linear strain regime. The shift of the damping properties also exhibits a linear relation between the damping factor $Q_{i}$ (Eq. 13) and the amplitude $A_{i}$ (Eq. 10) following Eq. 14 [8]: 


$$
\begin{aligned}
Q_{i} & =\frac{\tilde{f}_{i}}{\tilde{f}_{i, 2}-\tilde{f}_{i, 1}} \\
\frac{1}{Q_{i}}-\frac{1}{Q_{0}} & =\alpha_{Q}^{\mathrm{NIRAS}} \cdot A_{i}
\end{aligned}
$$

where $\tilde{f}_{i}$ is the resonance frequency of vibrations (Eq. 11), $\tilde{f}_{i, 1}$ and $\tilde{f}_{i, 2}$ are those frequencies on either side of the resonance at which the amplitude is $1 / \sqrt{2}$ times the amplitude at resonance, and $Q_{0}$ is the linear regime damping factor. For low excitation amplitudes, the frequency shift $\left(\tilde{f}_{0}-f_{\min }\right)$ is non-existent and the peak frequency and quality factor are considered to be obtained in the linear strain regime. In this study, $\tilde{f}_{0}$ and $Q_{0}$ are determined as the intersection with the $y$-axis of the linear relations between the peak amplitudes $A_{i}$ and the peak frequencies

${ }_{165} \tilde{f}_{i}$, and the peak amplitudes $A_{i}$ and the measured damping factors $Q_{i}$, respectively [22]. One expects to find an increase in the both parameters proportional to the hysteresis, $\alpha_{f}^{\text {NIRAS }}$ and $\alpha_{Q}^{\text {NIRAS }}$, with increasing damage of the specimen.

\subsection{NSIRAS}

A single-impact based version of the NIRAS technique was recently introduced by Eiras et al. [19]. In the so-called NSIRAS technique (Non-linear Single Impact Acoustic Spectroscopy), the reverberation from a standard impact resonance frequency test is analyzed using a short time Fourier Transform (STFT), and the non-linear frequency shift as a function of the amplitude can be successfully determined over a dynamic range. The method consists of a sliding window with fixed length $L, w^{L}(n)$, that moves in $S$-sample steps through a single reverberation signal, $y_{I}(n)$, and transforms the time segment within the $p$-window, $y_{I, w_{p}^{L}}(n)$, to the frequency domain at every temporal window position (Eq. 15).

$$
y_{I, w_{p}^{L}}(n)=y_{I}(n) \cdot w^{L}(n-p \cdot S), n \in\{0, \ldots, N-1\}, p \in\left\{0, \ldots,\left\lfloor\frac{N-L}{S}\right\rfloor\right\}
$$

The original study was done with a Tukey window, however, the mathematical formulation of this work has been done for any kind of window $w^{L}(n)$. In this study, a traditional rectangular window has been used (Eq. 16). The window length has been chosen to ensure that at least 10 periods in the fundamental ringdown signal are captured, the window shift $(S)$ is $30 \%$ of the window length, or a $70 \%$ overlap between consecutive windows.

$$
w^{L}(n)= \begin{cases}1, & 0 \leq n \leq L-1 \\ 0, & \text { otherwise }\end{cases}
$$

Figure 4 shows a schematic representation of the algorithm. The moving window analysis is stopped when the spectral amplitude falls below a preset threshold value (th), e.g. $5 \%$ of the maximum spectral amplitude measured in the first time window. Figure $4 \mathrm{~b}$ represents the frequency domain at every window position. It can be seen that the shape of the resulting amplitude spectra seem similar to the spectrum of a burst signal, and not as much 


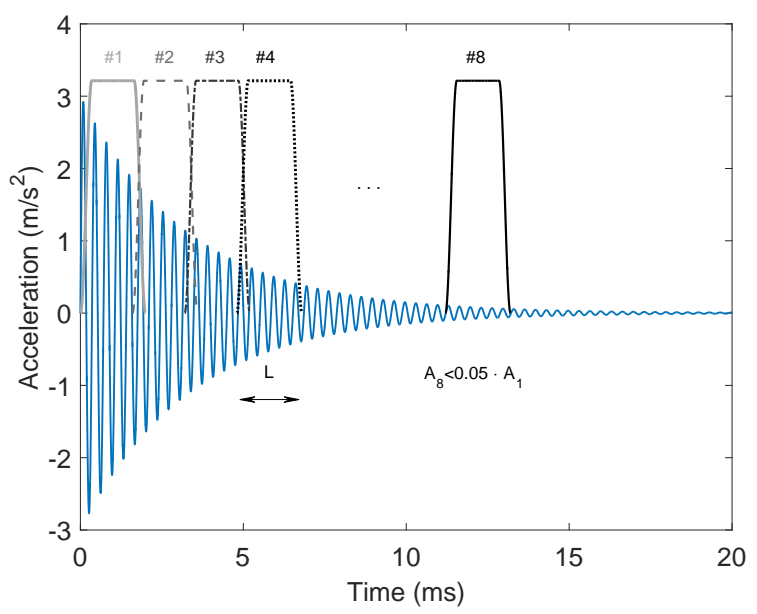

(a)

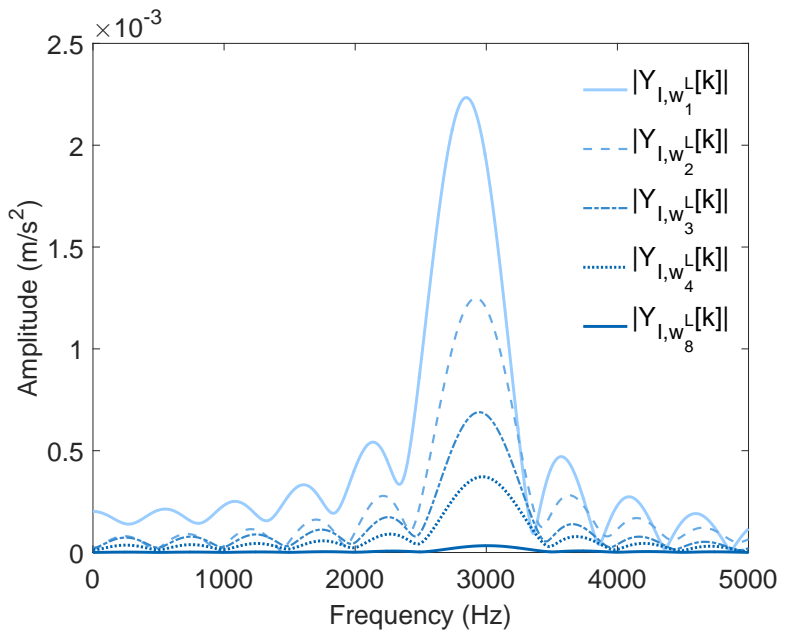

(b)

Figure 4: NSIRAS. a) Schematic representation of the signal processing algorithm to obtain the time-varying resonance frequency shift through the NSIRAS algorithm. b) Stacked frequency spectrum representation of the time signals within the first 8 windows.

to that of the reverberation signal seen in Figure 1 (red line). This windowing breaks the underlying effect of the exponentially decaying signal and the effects are reflected in the estimation of the non-linear parameters $\alpha_{f}^{\text {NSIRAS }}$ and $\alpha_{Q}^{\text {NSIRAS }}$. Both are estimated following the linear fit seen earlier; they are reformulated using the current variables according to the NSIRAS technique in Eqs 17, 18, 19, 20, and 21:

$$
\begin{aligned}
A_{I, p} & =\max \left\{\left|Y_{I, w_{p}^{L}}[f]\right|\right\} \\
\tilde{f}_{I, p} & =\max _{f}\left\{\left|Y_{I, w_{p}^{L}}[f]\right|\right\} \\
\frac{\tilde{f}_{I, 0}-\tilde{f}_{I, p}}{\tilde{f}_{I, 0}} & =\alpha_{f, I}^{\mathrm{NSIRAS}} \cdot A_{I, p} \\
Q_{I, p} & =\frac{\tilde{f}_{I, p}}{\tilde{f}_{I, p, 2}-\tilde{f}_{I, p, 1}} \\
\frac{1}{Q_{I, p}}-\frac{1}{Q_{I, 0}} & =\alpha_{Q, I}^{\mathrm{NSIRAS}} \cdot A_{I, p}
\end{aligned}
$$

where $\tilde{I}_{I, 0}$ and $Q_{I, 0}$ are the linear approximations for the linear regime resonance frequency and the damping factor, respectively, related to the reverberation signal $y_{I}(n)$.

\subsection{FANSIRAS}

The proposed technique, called Flipped Accumulative Non-Linear Single Impact Resonance Acoustic Spectroscopy (FANSIRAS), is based on the NSIRAS technique but with a variable length window. As with the spectrogram-based technique, the proposed method only requires a single reverberation signal $y_{I}(n)$ to obtain a reliable estimate of the non-linearity. The acronym of the technique corresponds to the original idea of the algorithm, based on the equivalent reconstruction of the signals of the NIRAS technique but from a single signal: 
flipping the signal and windowing cumulatively. In order to ease the mathematical nomenclature, the method is described as a window of initial length equal to that of the acquired signals, $N$, which progressively decreases its length to the lower bound, and transforms the time segment of the impact signal within the $p$ th window, $y_{I, w_{p}^{L_{p}}}(n)$, to the frequency domain at each window position as follows:

$$
y_{I, w_{p}^{L_{p}}}(n)=y_{I}(n) \cdot w^{L_{p}}(n-p \cdot M), \quad n \in\{0, \ldots, N-1\}, p \in\left\{0, \ldots,\left\lfloor\frac{N}{M}\right\rfloor-1\right\}
$$

Here, $w^{L_{p}}(n)$ represents the rectangular window, which shortens at each step of the algorithm. It has the same expression as in Eq. 16, but the length of the window varies at each position $p, L_{p}=N-p \cdot M . M$ represents the number of samples by which the window decreases at each step of the algorithm, and it is equivalent to the parameter $S$ of NSIRAS. It is related to the number of points used in the regression, but it does not significantly affect the estimation of the non-linear parameters. In this work, it is computed as the number of samples in 4 periods of the signal.

Figure 5a shows a schematic representation of the algorithm in the time domain. This algorithm preserves the phenomena underlying a reverberation signal, as can be seen in the resulting amplitude spectra (Figure 5b). Obviously, the first signal's amplitude spectrum coincides with the original input signal, since the whole signal is analyzed. As the time window shortens, the amplitude of the signals decrease and the resulting signals are equivalent to having excited the specimen with impacts of lesser strengths. As the strength of the impact decays, the peak amplitude decreases, and the resonance frequency increases. The damping factor also increases for each time window, as will be seen in the following (Section 2.3.1).

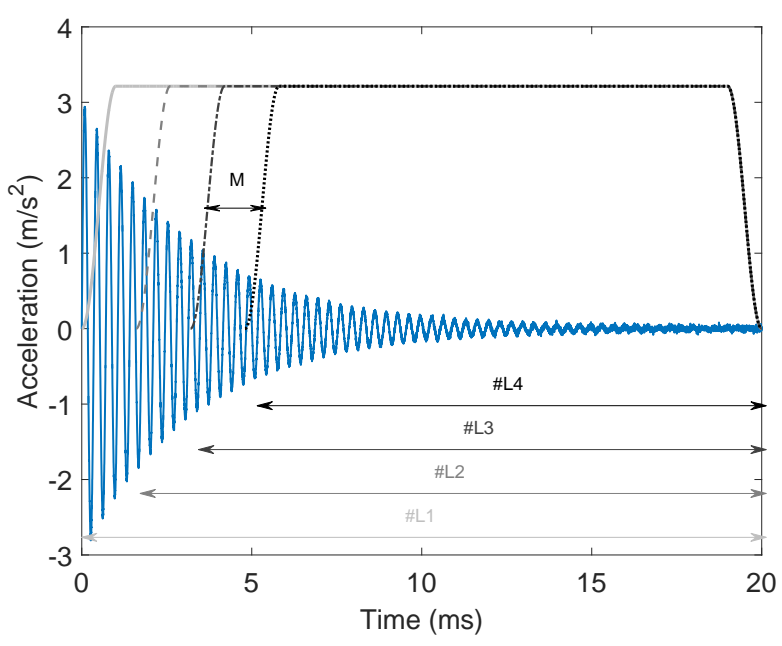

(a)

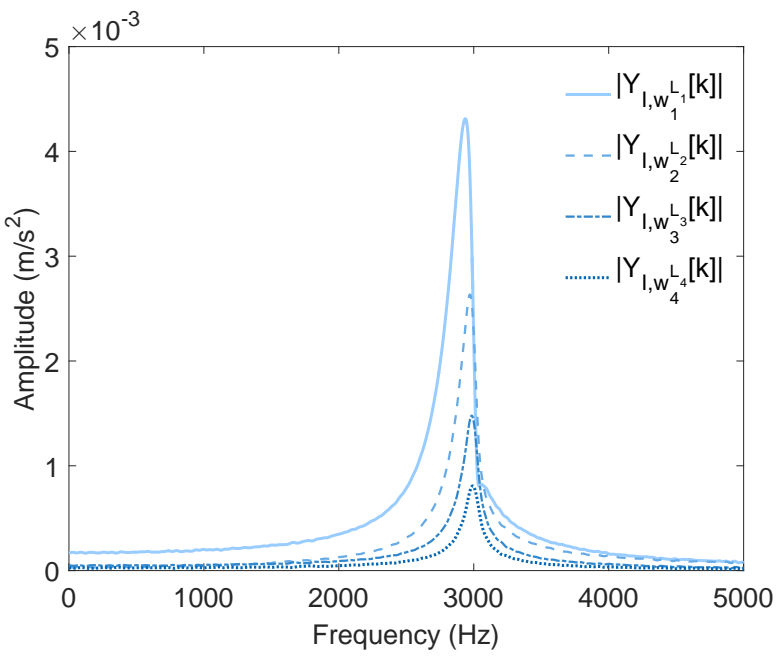

(b)

Figure 5: FANSIRAS. a) Schematic representation of the signal processing algorithm to obtain the time-varying resonance frequency shift through the FANSIRAS algorithm. b) Stacked frequency spectrum representation of the time signals within the first 4 windows.

The non-linearity parameters $\alpha_{f, I}^{\mathrm{FANSIRAS}}$ and $\alpha_{Q, I}^{\mathrm{FANSIRAS}}$ are both estimated with the previous linear fits according to the NSIRAS technique in Eqs 17, 18, 19, 20, and 21, but splitting the time signal with the new windowing 
approach (Eq. 22).

\subsubsection{Stop criterion}

Apart from being a feature related to the hysteresis behaviour of the material, the damping factor $1 / Q$ may reveal useful information for determining the final window in the FANSIRAS algorithm. The algorithm must stop before losing the signal information (too small window).

The progressive windowing done in the new proposed algorithm implies that the corresponding underlying chirp signal ranges from a larger minimum frequency up to the maximum linear frequency $\tilde{f}_{0}$. It supposes a lower bandwidth as $w^{L_{p}}(n)$ decreases its size. The mathematical expression of the damping factor controls the increasing of the peak frequency (which never stops growing) in the numerator split by the decreasing of the bandwidth as the window length decreases. What is used as the stop criterion is a comparison of the current value of the damping factor with the previous one. The window in the FANSIRAS algorithm is decreased until the value of $Q$ is lower than that obtained in the previous window.

Figure 6 represents an example of the amplitude spectrum obtained by the FANSIRAS algorithm applied to the simulated signal. The amplitude spectrum has been normalized in both axes: frequency, $\left(f-\tilde{f}_{I, p}\right)$, and amplitude, $A / A_{I, p}$. The large size of the bandwidth of the largest window can be observed, which implies the lowest value of the quality factor. The decreasing window in the FANSIRAS algorithm is decreased until there occurs a value of $Q$ lower than the previous window. In the example, the selected number of different time windows applied in the FANSIRAS algorithm would be 8 .

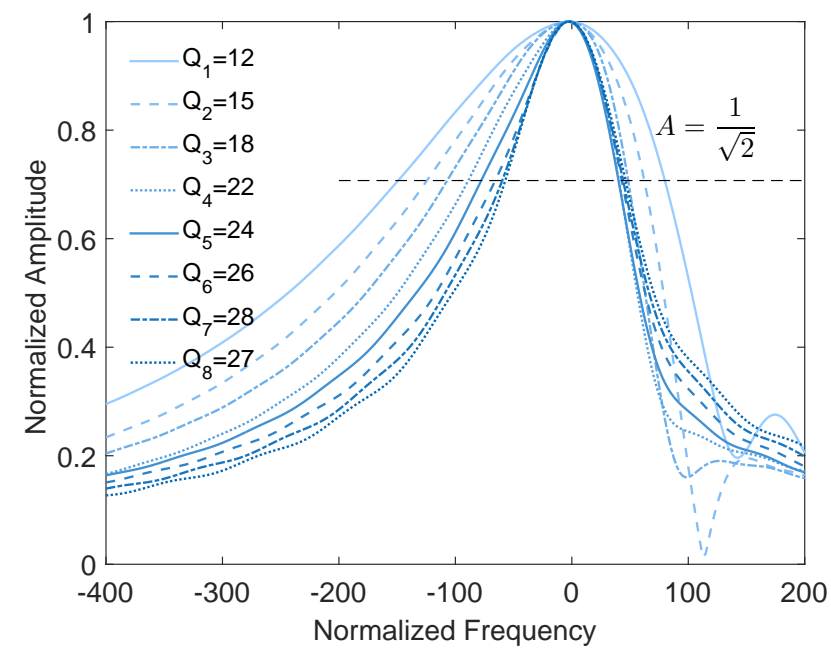

Figure 6: Stop criterion in the FANSIRAS algorithm. Example of the amplitude spectra obtained for consecutive windows by FANSIRAS algorithm applied to a real signal. The decreasing window is decreased until there occurs a value of $Q$ lower than the previous window.

In the following, the non-linearity parameters for NSIRAS are referred to as $\alpha_{f}^{\text {NSIRAS }}$ and $\alpha_{Q}^{\text {NSIRAS }}$, and those for the FANSIRAS algorithm as $\alpha_{f}^{\text {FANSIRAS }}$ and $\alpha_{Q}^{\text {FANSIRAS }}$, taking into account that they are extracted from the largest impact reverberation signal in each experience. 


\subsection{Comparison}

In order to fully understand the effects of the windowing on the estimation of the non-linear hysteretic parameters, the results of NIRAS, NSIRAS and FANSIRAS algorithms applied on the simulation signal raised in Figure 1 are compared in Figure 7. The blue line represents the reference strongest original signal spectrum obtained from the simulated model seen in Figure 3; the NSIRAS and FANSIRAS results are obtained from it. The green lines represent the first 8 NSIRAS stacked spectra and the red lines represent the 8 first FANSIRAS spectra. This representation allows the comparison of how the resonant frequencies migrate with time and amplitude for the same measured signal depending on the applied algorithm. The short-time frequency transform (STFT) done in the NSIRAS algorithm results in a down-biased amplitude estimation. The STFT also avoids the weighted average of the whole frequency content available in the signal, allowing a ranging more widely over frequencies. The interval of frequencies ranges from a lower minimum resonance frequency, $f_{\min }$, up to the same $f_{\max }$ than NIRAS. The new proposed FANSIRAS algorithm is able to extract equivalent amplitude spectra to the traditional NIRAS technique from a single signal. The decreasing cumulative window respects not only the decreasing exponential shape but also the frequency average along the whole ring-down.

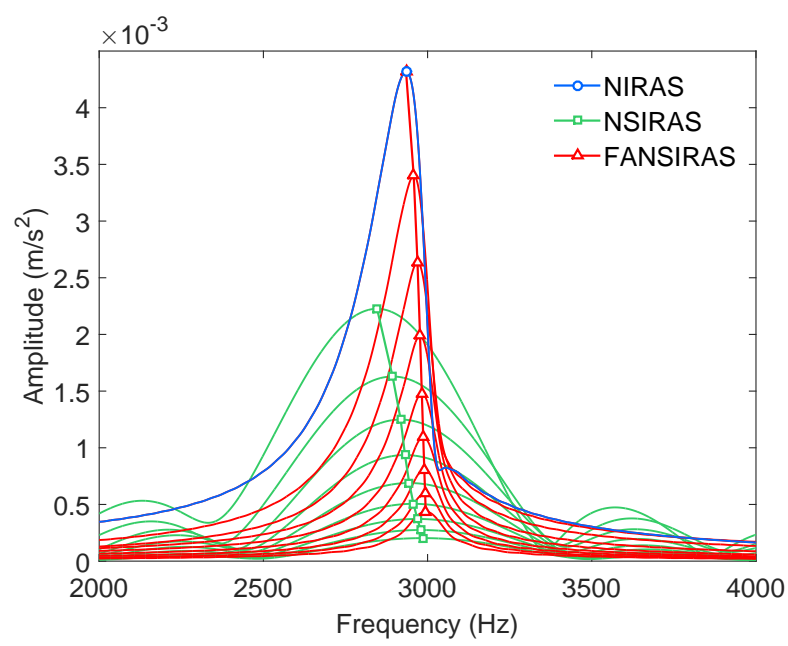

Figure 7: Comparison of the simulated signal amplitude spectrum computed by NSIRAS and FANSIRAS algorithms. The blue line represents the reference original simulated spectrum, the green lines represent the first 8 NSIRAS stacked spectra and the red lines represent the 8 first FANSIRAS spectra. a smaller amplitude interval. It results in a significantly higher value of slope $\alpha_{f}^{\text {NSIRAS }}$ than $\alpha_{f}^{\text {NIRAS }}$ for the same 


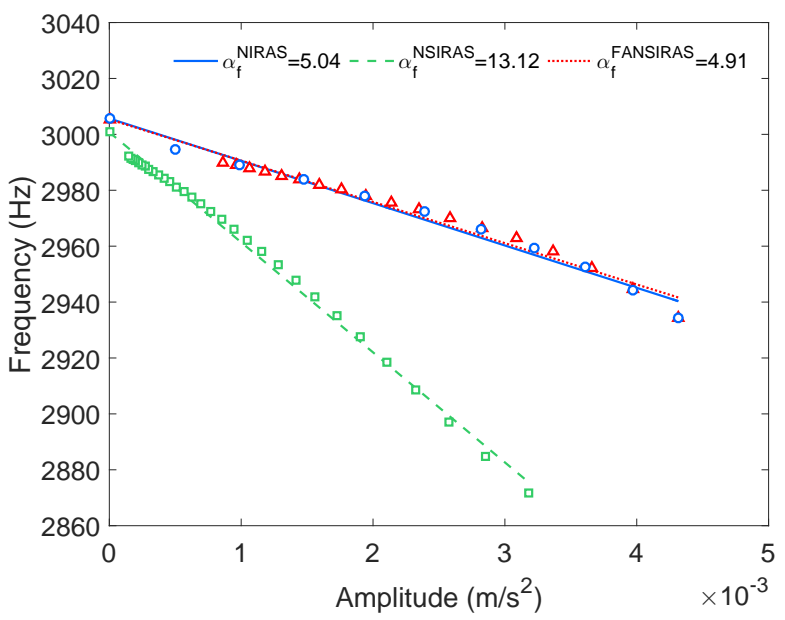

(a)

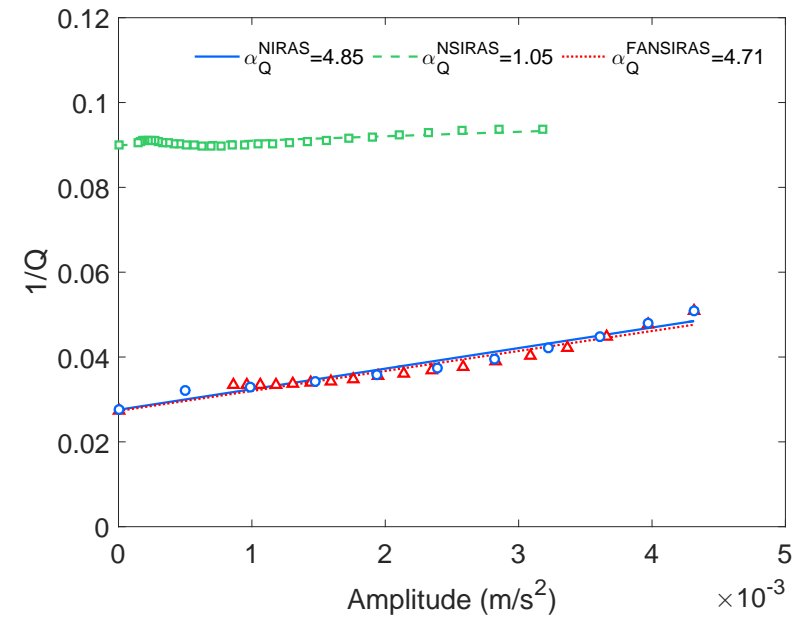

(b)

Figure 8: Comparison of NIRAS, NSIRAS and FANSIRAS algorithms for a simulated signal. a) Amplitude dependent resonance frequency shift computation $\left(\alpha_{f}\right)$. b) Amplitude dependent resonance damping shift computation $\left(\alpha_{Q}\right)$. The y-axis interceptions represent the values $\tilde{f}_{0}$ and $Q_{0}$ for each technique, respectively.

simulated model. The parameter $\alpha_{f}^{\text {FANSIRAS }}$ results equivalent to $\alpha_{f}^{\text {NIRAS }}$. Therefore, the new windowing approach demonstrate a better estimation than the original single-based technique NSIRAS, at least, for the proposed numerical model.

As far as the authors' knowledge extends, the study of the variation of the amplitude-dependent damping had never been done before for the NSIRAS technique. It has been included in this study in order to show the importance of the kind of windowing when studying the quality factor of a reverberation signal. Figure 8b compares the results obtained by NIRAS and NSIRAS. At a glance, it can be observed that the $\alpha_{Q}^{\text {NSIRAS }}$ values are greater and less varying than $\alpha_{Q}^{\text {NIRAS }}$. Although NSIRAS is able to estimate the shift of the peak frequency, the shape of the resulting time signals are almost equivalent to pure sinusoidal signals with an almost constant bandwidth bigger than in the NIRAS technique, proportional to the length of the window. Figure $8 \mathrm{~b}$ also compares the variation of the damping factor obtained both from the NIRAS and FANSIRAS techniques. At a glance, it can be observed that FANSIRAS algorithm allows estimating the damping values equivalently to the NIRAS algorithm but from a single signal.

The shortening of the time signal from the lower bound results on two different effects over the two involved signals. For the instantaneous amplitude, $a[n]$, it allows reducing the amplitude of the signal $a$ but keeping constant the exponential decay factor $\gamma$. For the time-varying signal, $s[n]$, the decrease in the temporal window amounts to a narrower bandwidth $(\Delta f)$. Therefore, the proposed numerical model (later verified with real experiments) shows that the variation of the damping factor $Q$ with the amplitude is exclusively sensitive to changes on the bandwidth. There is not an amplitude-dependent attenuation. According to that, it must be noted that for the NIRAS and FANSIRAS algorithms applied over the theoretical model, the parameters of $\alpha_{f}$ and $\alpha_{Q}$ have resulted on similar numerical values. Despite having been computed by different procedures they are 
sensitive to the same effect: the widening of the underlying exponential phase-varying signal as the amplitude increases. In further sections, both hysteretic parameters for the three raised techniques are compared for a real dataset.

\section{Experimental}

\subsection{Materials and specimens}

Three mixes of standardized Portland cement mortar (water/cement $=0.5$ ) made of 450 grams of Spanish cement CEM I-52.5-R (supplied by Cementval, Puerto de Sagunto, Spain), 1350 grams of 0/2 crushed quartz

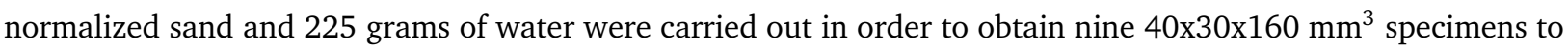
perform the present study. This geometry was chosen with the intention of avoiding two fundamental flexural modes in close frequencies coming from imperfections in the mixing and manufacturing process. Having a square cross section stimulates modal coupling between two resonance modes with similar frequencies, resulting in peak splitting in the spectra, and consequently, a decrease of accuracy in the frequency analysis. After the mixing process, filled iron moulds were stored in a wet chamber $\left(20^{\circ} \mathrm{C}\right.$ and $100 \%$ H.R.) for 24 hours, then the nine mortar pieces were released and stored in the wet chamber again for 60 days in order to reach the stabilization stage of elastic properties. Afterwards, the specimens were dried to constant mass in an oven at $40^{\circ} \mathrm{C}$ for 7 days and wrapped with plastic film until they reached room temperature again. These prismatic specimens were characterized by resonance impact acoustic spectroscopy, in order to determine their properties in the pristine state (TH40). Two thermal damage treatments were performed according to the chemical decomposition of Portland cement hydrates. First, the pristine specimens were thermally damaged at $400^{\circ} \mathrm{C}$, achieving the decomposition of the calcium silicate hydrates (C-S-H) and calcium aluminate (C-A-H) and sulphoaluminate (Ettringite, monosulphate) hydrates, reaching this temperature in two hours, maintaining it for three hours, and slowly cooling for twelve hours inside the oven (TH400). Once room temperature was reached, the mortar bars were tested again by impact resonance acoustic spectroscopy. Second, the $400^{\circ} \mathrm{C}$-treated specimens were thermally damaged at $525^{\circ} \mathrm{C}$ (this favours the $\mathrm{Ca}(\mathrm{OH})_{2}$ decomposition) following the same heating-cooling procedure as explained above (TH525). This way, the same 9 specimens had been characterised for the three different treatments: TH40, TH400 and TH525.

\subsection{Test layout}

Acoustic resonance tests were performed with the elements as shown in Figure 9. An impact hammer (Brüel \& Kjær 8206-003) was attached to an axis, located in a metallic structure. This axis, supported on two ball bearings, yields a free rotational motion in one plane with minimum friction. The specimen was placed on the metallic supports, slightly sloping, positioned for impact in the centre of the face and perpendicular to the axis of greater inertia of its cross section. In the opposite top-left corner, a piezoelectric accelerometer sensor (PCB 352A21, $0.956 \mathrm{mV} / \mathrm{m} / \mathrm{s}^{2}$ sensitivity) was attached in order to obtain the vibrational motion of the test probe. The electric 


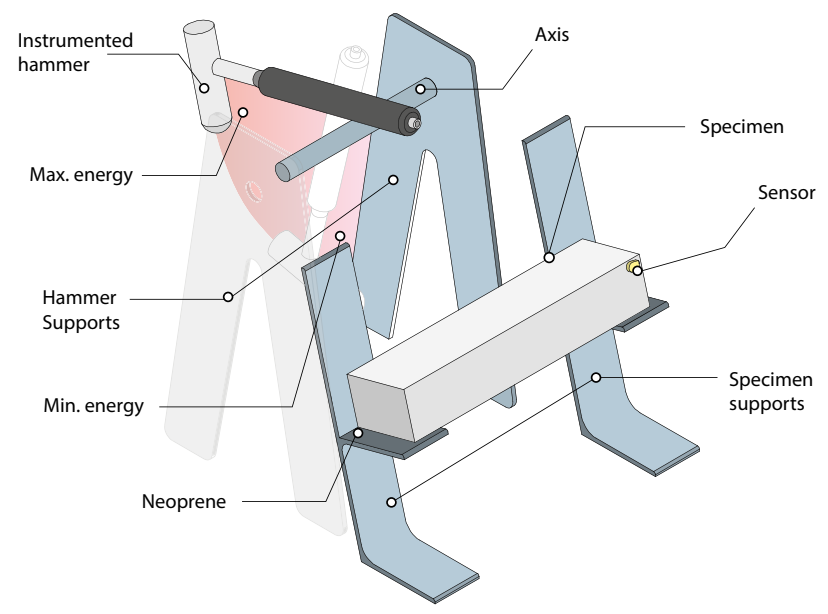

Figure 9: Experimental layout of the support hammer, disposition of the specimen, and location of the sensor.

signal of the instrumented hammer and the accelerometer go through a signal conditioner (PCB 482A18), so as to be polarized and then transmitted to the oscilloscope (Tektronics MDO3014). The oscilloscope was configured to optimize the signal acquisition and processing with $f_{s}$ equal to $250 \mathrm{kHz}, N$ equal to 10000 samples, trigger voltage equal to $120 \mathrm{mV}$, and pre-trigger time equal to $4 \mathrm{~ms}$. Finally, the information was transmitted via USB to a computer with a control software code developed by the authors. Taking advantage of the potential offered by the Instrument Control Toolbox of the MATLAB software package, a graphical user interface was developed, for the automation of the technique and to optimize it with respect to the test procedure requirements.

\subsection{Reproducibility test}

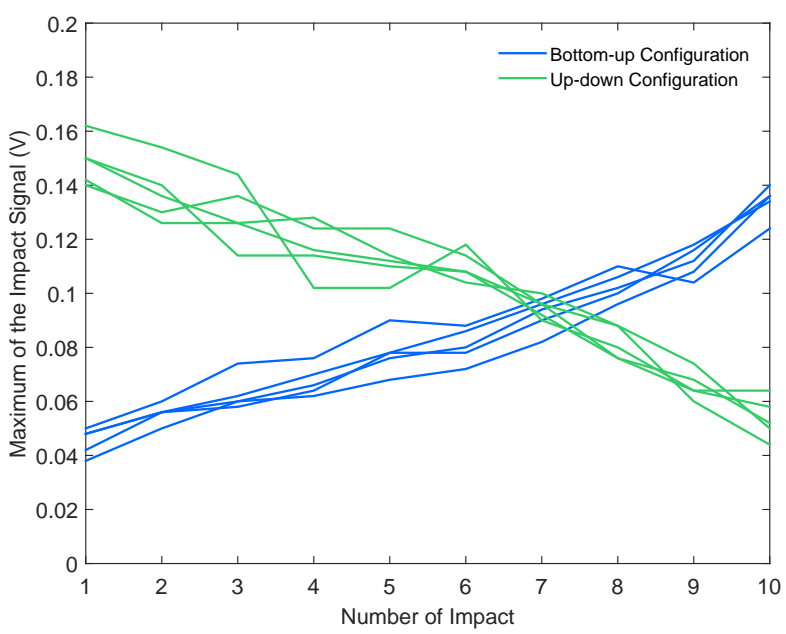

(a)

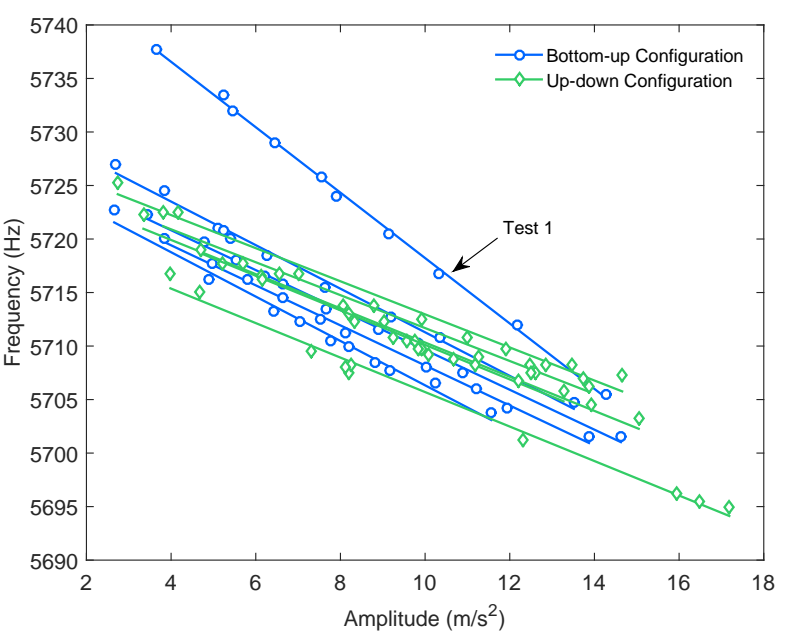

(b)

Figure 10: Reproducibility Test. Five repetitions of the resonance test (10 different amplitude impacts) in the same specimen in bottom-up (starting with the lowest impact) configuration (blue) and up-down (starting with the highest impact) configuration (green) were carried out. 10a Maximum amplitude of the impact signal. 10b Relation between the spectral peak amplitude and the resonance frequency. The markers represent the experimental results and the straight lines represent the best fitted linear model for each test. 
In order to assess the robustness of the techniques, a reproducibility test of the non-linear resonance technique was performed (10 impacts of different energies were applied), making 5 repetitions in the same specimen in the bottom-up (starting with the lowest impact) configuration and the up-down (starting with the highest impact) configuration. The purpose of testing two different configurations is to make sure the discrete memory of the material does not affect the calculation of the non-linearity parameters [3, 23]. For this reason, there was a 24 hour space of time between the tests in the bottom-up configuration and those in the up-down configuration. In Figure 10a there can be seen the maximum intensity in the impact curve described by the instrumented hammer. In this graph, the repeatability of the impact energy can be seen since the 5 repetitions are in the same range and magnitude for all impact levels and both configurations. Figure 10b shows the peak amplitude spectrum versus peak frequency plot for the 5 tests made in each configuration. A very significant difference between the correlation from the first test in the bottom-up series and the rest of the correlations can be appreciated. This behaviour matches some experiments previously reported, when a specimen of mesoscopic material is conditioned with an external source of motion and it takes several hours to recover the mechanical properties of its initial state $[5,24]$. Hence, the authors decided undertaking the experiment in the up-down configuration to ensure the reproducibility of the test and perform a proper comparison of the different procedures for assessing the non-linearity parameters.

\section{Results and Discussion}

Figure 11 shows representative resonance spectra obtained at different impact energies for one mortar sample at the different temperatures. It is important to notice how these real spectra fit to the model showed in Section 2. In the first instance, the TH40 specimens exhibited good performance due to the fact that the evaporated water inside the porous structure of the material had no influence on the material's mechanical properties. The second thermal treatment, the TH400 series, forced the evaporation of part of the water combined in the S-C-H, sulphoaluminates, and the A-C-H of the hydrated Portland cement, yielding a loss of stiffening and increasing the non-linearity of the system. Lastly, the TH525 series were severely damaged, greatly increasing the non-linearity and losing mechanical performance. The described damage produced by high temperatures resulted in 4 effects that can be distinguished in the spectra presented here: a progressive decrease of the resonance frequency, an increase of signal bandwidth, a downward shift of the resonance frequency with increasing impact energy, and the resonance peak became noticeably asymmetric. Although the impact energy was the same for all series and specimens, the maximum intensity reached in the spectra decreased as the damage in the specimens increased.

\subsection{NIRAS}

350

To evaluate the thermal damage in the mortar specimens, the hysteretic non-linearity parameters $\alpha_{f}^{\mathrm{NIRAS}}$ and $\alpha_{Q}^{\text {NIRAS }}$ were computed on the basis of the NIRAS technique described in Section 2.1. Representative results of 


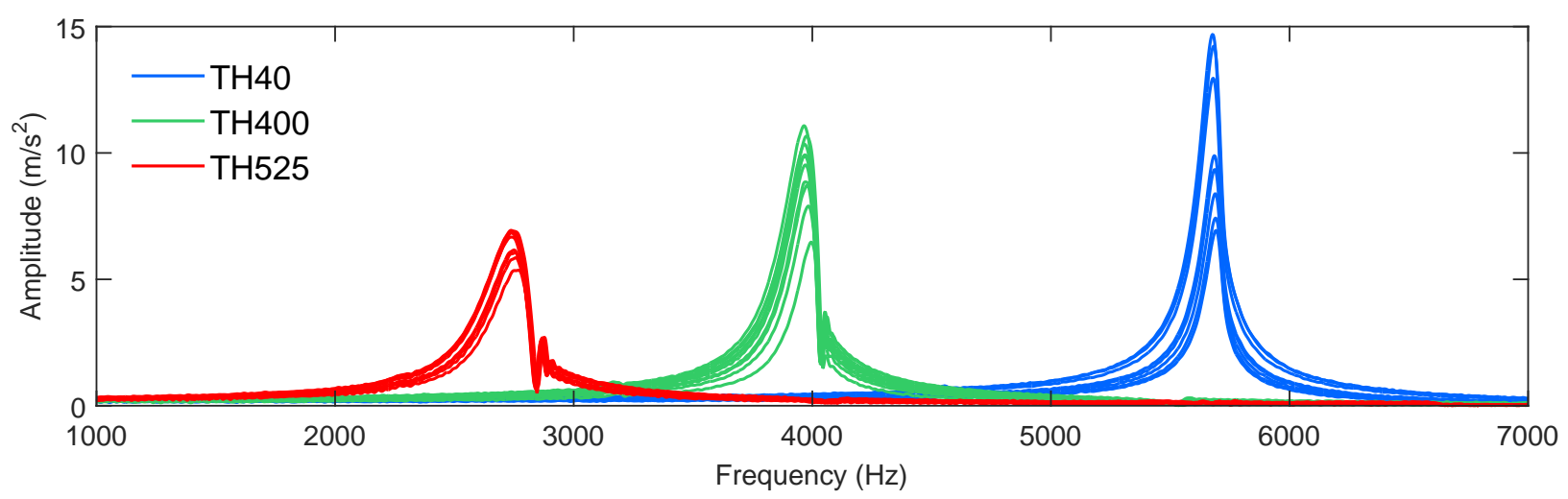

Figure 11: Representative resonance spectra obtained at different impact energies for one mortar sample at the temperatures $40^{\circ} \mathrm{C}$ (blue line), $400^{\circ} \mathrm{C}$ (green line) and $525^{\circ} \mathrm{C}$ (red line).

the amplitude-dependent dynamic features (resonance frequency and damping) at different temperatures $\left(40^{\circ} \mathrm{C}\right.$, $400^{\circ} \mathrm{C}$ and $525^{\circ} \mathrm{C}$ ) are shown in Figure 12 .

Figure 12a shows the difference in resonance frequencies versus the peak of the spectral amplitude. The linear regime frequency $\tilde{f}_{0}$ is the amplitude-independent resonance frequency computed in the linear regime $(A=0)$. The parameter $\alpha_{f}^{\text {NIRAS }}$ of the thermally damaged concrete was determined by the slope of the linear regressions (solid lines) of the 10 plotted results (hollow circles), which rapidly increases with the damaging temperature. Figure $12 \mathrm{~b}$ shows the evolution of the damping as a function of the spectral amplitude, at different damage states. The linear damping factor $Q_{0}$ is also computed in the linear strain stage $(A=0)$. These results demonstrate that both hysteretic parameters are sensitive to the internal damage of the material caused by the thermal treatment, and that shifts of resonance frequency are paired with shifts in $Q$ values.

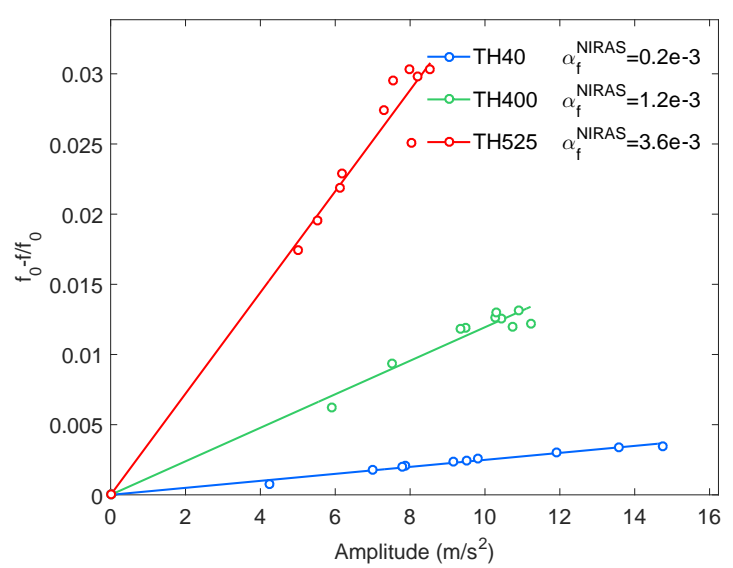

(a)

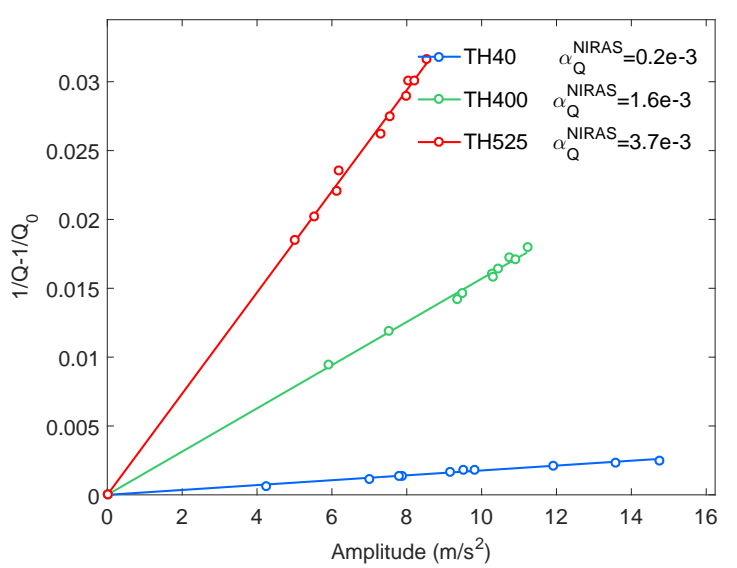

(b)

Figure 12: NIRAS. Representative results of the amplitude-dependent dynamic features at different temperatures $40^{\circ} \mathrm{C}$ (blue), $400^{\circ} \mathrm{C}$ (green) and $525^{\circ} \mathrm{C}$ (red) computed by the NIRAS technique. The markers represent the experimental results and the straight lines represent the best fitted linear model. a) Resonance frequency shift ( $\left.\alpha_{f}^{\text {NIRAS }}\right)$. b) Attenuation ( $\left.\alpha_{Q}^{\text {NIRAS }}\right)$. 


\subsection{NSIRAS}

Unlike the NIRAS technique, the NSIRAS method investigates the non-linearity of the material using a single impact event, which allows reducing the total number of impacts. Figure 13 compares the variations in resonance

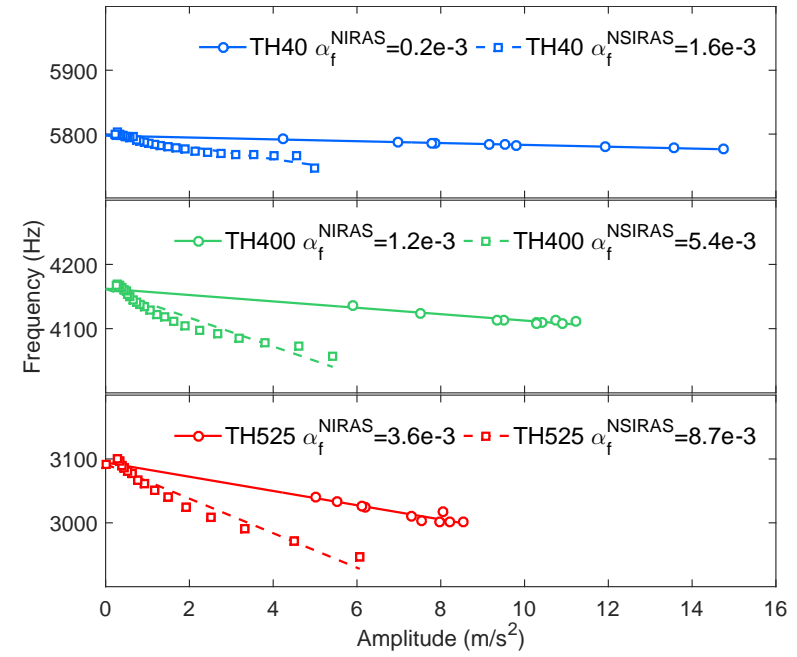

(a)

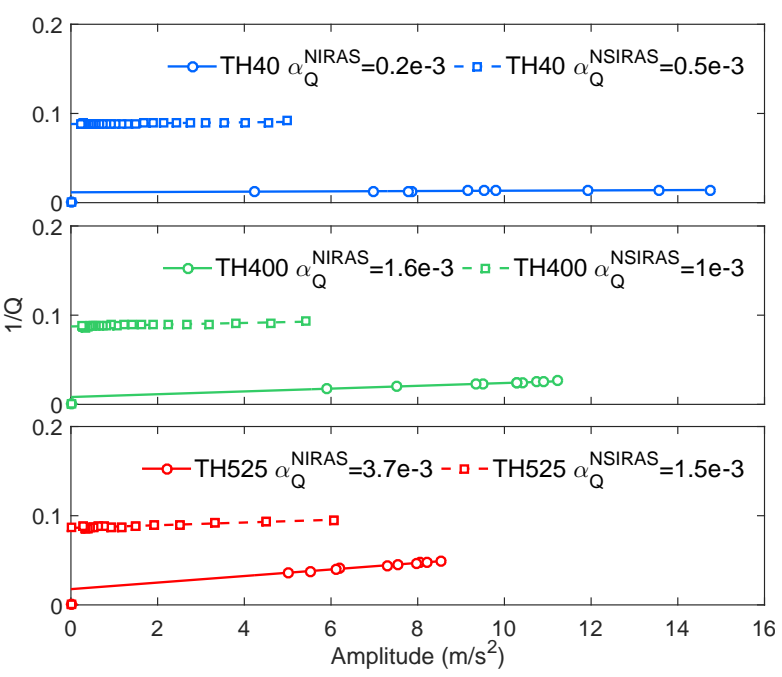

(b)

Figure 13: NSIRAS. Comparison of representative results for the amplitude-dependent dynamic features at different temperatures $40^{\circ} \mathrm{C}$ (blue), $400^{\circ} \mathrm{C}$ (green) and $525^{\circ} \mathrm{C}$ (red) computed by NIRAS (solid lines) and NSIRAS (dashed lines). The markers represent the experimental results and the straight lines represent the best fitted linear model. 13a Resonance frequency shift ( $\alpha_{f}^{\text {NIRAS }}$ and $\left.\alpha_{f}^{\text {NSIRAS }}\right)$. 13b Attenuation ( $\alpha_{Q}^{\text {NIRAS }}$ and $\left.\alpha_{Q}^{\text {NSIRAS }}\right)$.

As seen for the theoretical model (Section 2.4), the STFT applied in the NSIRAS algorithm significantly reduces the amplitude estimation, while avoids the weighted average of the whole frequency content. It results on higher values of the linear slope $\alpha_{f}^{\text {NSIRAS }}$ than $\alpha_{f}^{\text {NIRAS }}$. Moreover, the relation obtained from the NSIRAS algorithm between the peak amplitude values and the estimated frequencies is rather non-linear. Whereas the undamaged specimen still exhibited a fairly linear behaviour (like in the simulated model), an important deviation from the linear model is especially notable for damaged samples. According to this study, this might be due to the differences between the characteristic exponentially decaying envelope of the signal (Eq. 6) and the also exponential frequency variation (Eq. 2). The difference are more pronounced as the level of damage of the material increases.

Figure 13b compares the results obtained by NIRAS and NSIRAS for the amplitude-dependent damping factor. As occurred with the theoretical analysis, it can be observed that the $\alpha_{Q}^{\mathrm{NSIRAS}}$ values are greater and less varying 
than $\alpha_{Q}^{\text {NIRAS }}$ due to the STFT analysis. The variable $\alpha_{Q}^{\text {NSIRAS }}$ increases with the damage although less noticeably than that observed from the NIRAS study.

Lastly, it must be noted that this processing approach is very sensitive to the input parameters (window length) and its relation with the reverberation signal under study $\left(f_{0} / f_{s}\right)$. A bigger window length implies lower frequency variations but greater attenuation variations due to the averaging of the frequency content in the temporal window and the corresponding bandwidth. This implies that similar studies which determine the non-linear parameters can not be compared if the number of periods of the signal per window is not the same.

\subsection{FANSIRAS}

Like the NSIRAS technique, the new proposed FANSIRAS algorithm analyses the hysteretic behaviour of the material from the reverberation signal coming from the strongest strength impact. Figure 14 compares some representative results of the reference NIRAS technique and the new proposed FANSIRAS technique. The parameters used in the FANSIRAS algorithm were a window with initial length equal to the acquired signals, $N$ equal to 10000 samples, which progressively decreases by 400 samples until reaching a window with a quality factor lower than the previous one.

Figure 14a plots the relation between the resonance frequency and the spectral peak amplitude, and the best fitted linear model in every damage state. The starting point of the regression (highest amplitude values) obviously coincides for both techniques since the analyzed signals are the same. The linear regressions are equivalent, but the FANSIRAS algorithm evolves to values of lower amplitudes and higher linear frequencies along the ringdown of the signal. This effect supposes a slight upwards bias of the slope of the estimated linear regression, as will be seen later, and this is more pronounced as the damage of the material increases. This means that the new proposed approach slightly overcomes the drawback to the analytical procedure present in the NIRAS algorithm technique, and previously noticed in [20]. It was pointed out that the frequency shift may be evaluated in a relatively limited dynamic range of amplitude values. FANSIRAS gets to increase the measurable dynamic range from smaller peak amplitudes to the same largest value, which improves the sensitivity when estimating the linear regime parameters $\left(\tilde{f}_{0}\right.$ and $\left.Q_{0}\right)$.

Figure 14b compares the variation of the damping obtained both from the NIRAS and FANSIRAS techniques. It can be observed that FANSIRAS algorithm allows estimating the damping values equivalently to the NIRAS algorithm from a single signal also for real signals. The hysteretic parameter $\alpha_{Q}^{\text {FANSIRAS }}$ clearly classifies the level of damage following the variations of the damping factor.

\subsection{Comparison}

In this study, it has been suggested that the single signal based algorithms, NSIRAS and FANSIRAS, have to be applied to the signals from the impact of greatest strength in order to ensure that the peak frequency variable ranges up to its maximum value. Figure 15 compares the evolution of the non-linear hysteretic features, $\alpha_{f}$ and $\alpha_{Q}$, for each technique (NIRAS, NSIRAS and FANSIRAS) and each impact strength on a representative example at 


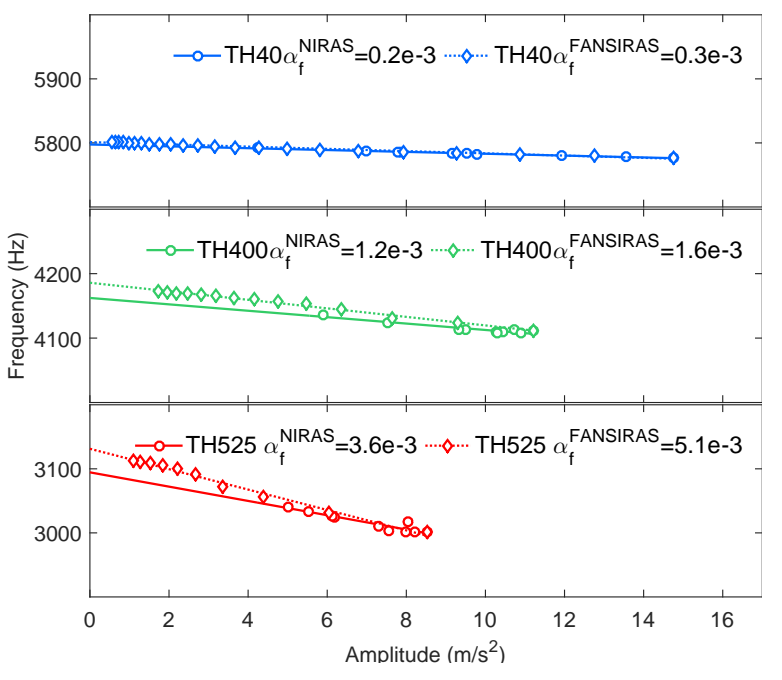

(a)

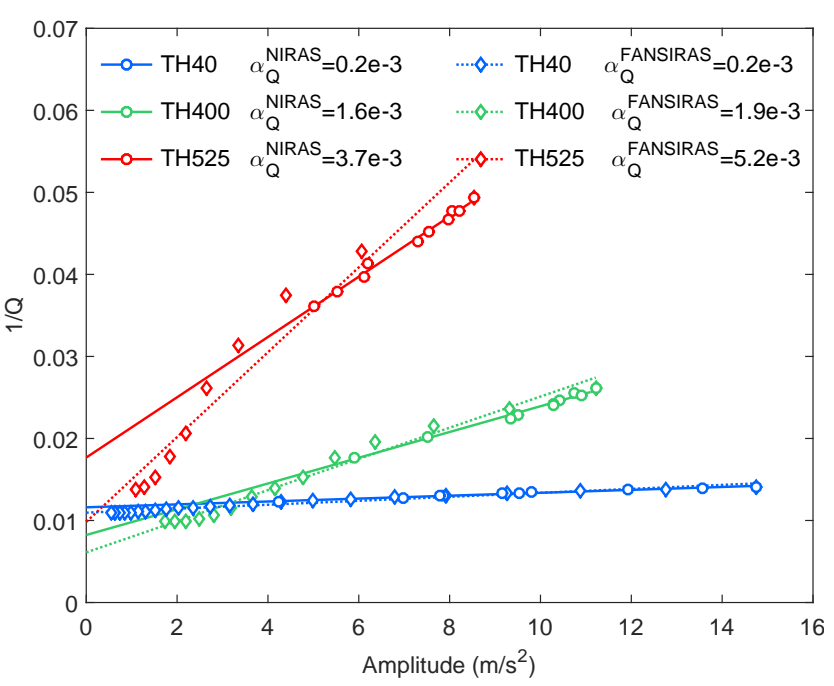

(b)

Figure 14: FANSIRAS. Comparison of representative results of the amplitude-dependent dynamic features at different temperatures $40^{\circ} \mathrm{C}$ (blue), $400^{\circ} \mathrm{C}$ (green) and $525^{\circ} \mathrm{C}$ (red) computed by NIRAS (solid lines) and FANSIRAS (dotted lines). The markers represent the experimental results and the straight lines represent the best fitted linear model. a) Resonance frequency shift ( $\alpha_{f}^{\mathrm{NIRAS}}$ and $\alpha_{f}^{\mathrm{FANSIRAS}}$ ). b) Attenuation ( $\alpha_{Q}^{\mathrm{NIRAS}}$ and $\alpha_{Q}^{\mathrm{FANSIRAS}}$ ).

the different studied levels of damage. In each plot, $\alpha_{f}^{\text {NIRAS }}$ and $\alpha_{Q}^{\text {NIRAS }}$ are the constant reference values obtained from the 10 impact signals. For NSIRAS (striped lines) and FANSIRAS (dotted lines), different results are obtained for each impact signal level. For the three analyzed levels of damage, $\alpha_{f}^{\text {NSIRAS }}$ and $\alpha_{Q}^{\text {NSIRAS }}$ yield closer values to the reference results for higher levels of impact. However, the results obtained by the new proposed technique, $\alpha_{f}^{\text {FANSIRAS }}$ and $\alpha_{Q}^{\text {FANSIRAS }}$, seemed to be more robust against the different impacts.

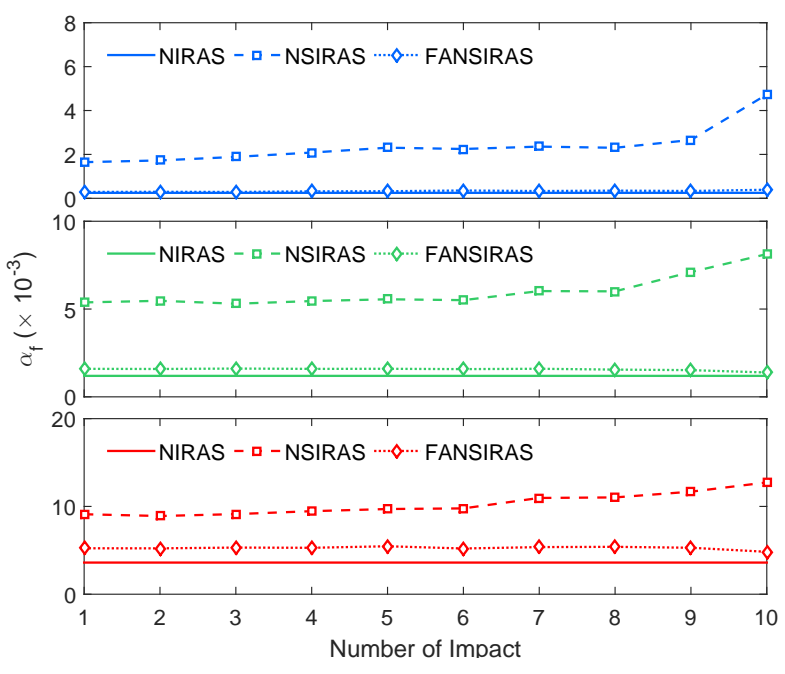

(a)

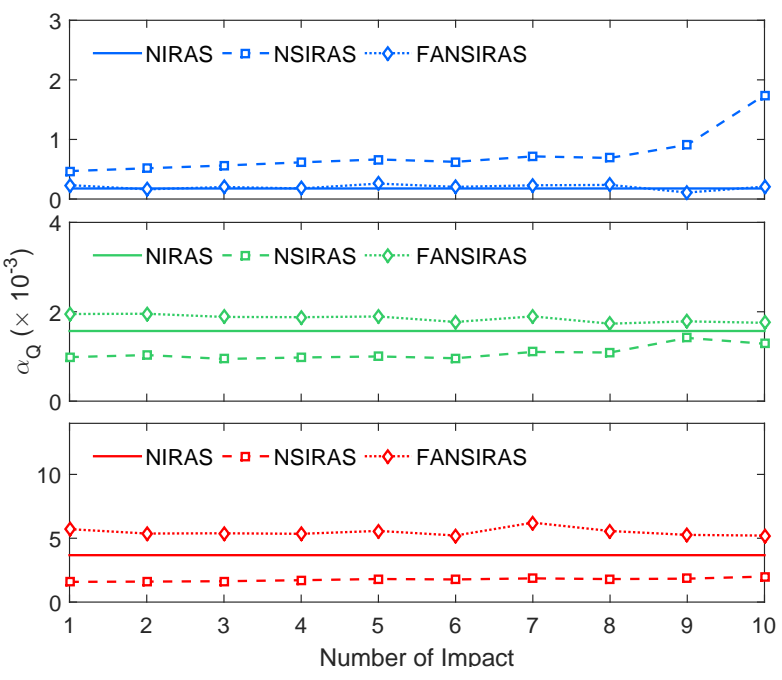

(b)

Figure 15: Comparison of the evolution of the non-linear hysteretic features for each technique, NIRAS (solid lines), NSIRAS (striped lines) and FANSIRAS (dotted lines), and each impact strength, on a representative example at the different studied levels of damage, $40^{\circ} \mathrm{C} \mathrm{(blue),} 400^{\circ} \mathrm{C}$ (green) and $525^{\circ} \mathrm{C}$ (red). a) $\alpha_{f}$, b) $\alpha_{Q}$.

Resonance tests were performed on all the mortar pieces fabricated (9 specimens) to ensure the reproducibility 
of the hysteretic measurements. The robustness test results are shown in Figure 16, and they confirm that the non-linear resonance vibration tests can provide a consistent measure of the internal damage of the specimens. Particularly for each studied technique, the resulting trends coincides with the results seen both for the simulated model (Section 2.4) and for the representative example described in previous Sections 4.1, 4.2, 4.3. The NIRAS technique is considered to be the reference result. The previously proposed NSIRAS technique is biased upwards when measuring the frequency shift $\left(\alpha_{f}\right)$ and significantly down biased when measuring the amplitude-dependent damping factor. The brand-new technique FANSIRAS coincides with the NIRAS results for undamaged results. However, they are slightly up biased when damage is present. This trend has previously been justified due to the analysis done by FANSIRAS up to lower amplitude values along the ring down.

Because the linear parameters $\tilde{f}_{0}$ and $Q_{0}$ are estimated independently of the value of the amplitude of the weaker impact coup, the hysteretic parameters $\alpha_{f}$ and $\alpha_{Q}$ are equivalent for both the NIRAS and FANSIRAS techniques. This is a result that has never before been diffused, as far as the authors' knowledge extends, but which is explained since both features are proportional to the same underlying hysteric behaviour of the material.

435 Figure 17 plots the root mean square error (RMSE) computed for all the experimental results versus the obtained linear fits. Whereas for undamaged specimens the regressions still exhibit fairly linear relations, the relations become rather non-linear for the damaged specimen tests. This behaviour is closely correlated to the need to use higher order coefficients in Eq. 1. Particularly, the high value of $\alpha_{Q}^{\text {FANSIRAS }}$ for TH525 is due to its characteristic S-shape data distribution as the level of damage increases (Figure 14b). The characteristic S-shape is directly

${ }_{440}$ attributed to $Q$ parameter estimation: the difference appears when computing the lowest frequency bound, $f_{1}$ (Eq. 20), closely related to the stiffness of the material for the highest amplitude deformations. This difference might be related to the nature of the signals used in each spectroscopy technique. NIRAS procedure estimates non-linear hysteretic parameters from original and several reverberation signals, however, FANSIRAS algorithm estimates the different strain levels from a single relaxing signal. The fact of $f_{1}$ being in the denominator might 445 boost the S-shape trajectory. 


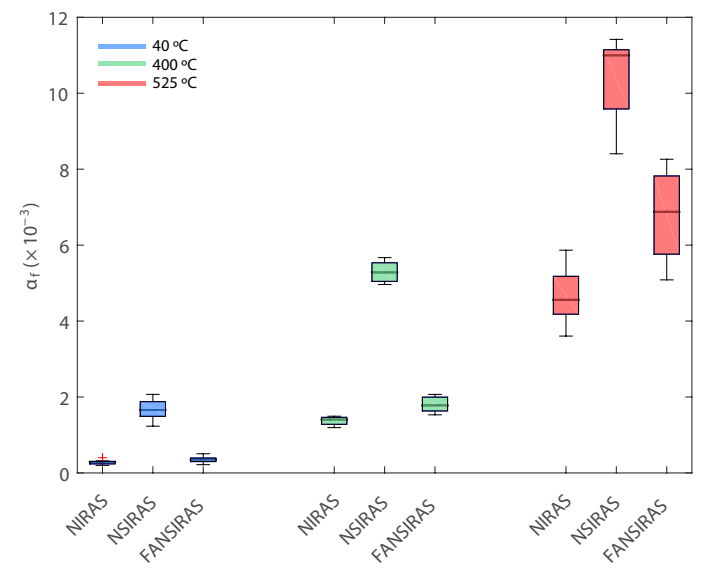

(a)

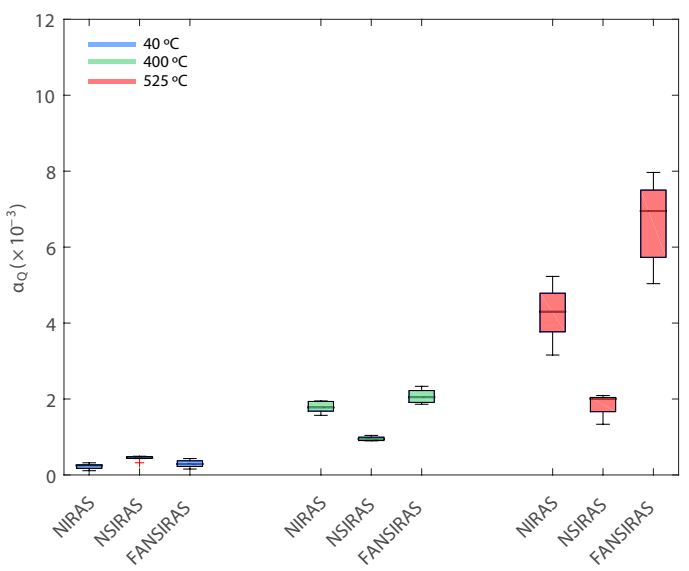

(b)

Figure 16: Comparing boxplots of the results of the amplitude-dependent dynamic features at different temperatures $40^{\circ} \mathrm{C}$ (blue), $400^{\circ} \mathrm{C}$ (green) and $525^{\circ} \mathrm{C}$ (red) computed by the different analyzed techniques: NIRAS, NSIRAS and FANSIRAS. a) Resonance frequency shift $\left(\alpha_{f}\right)$. b) Attenuation $\left(\alpha_{Q}\right)$.

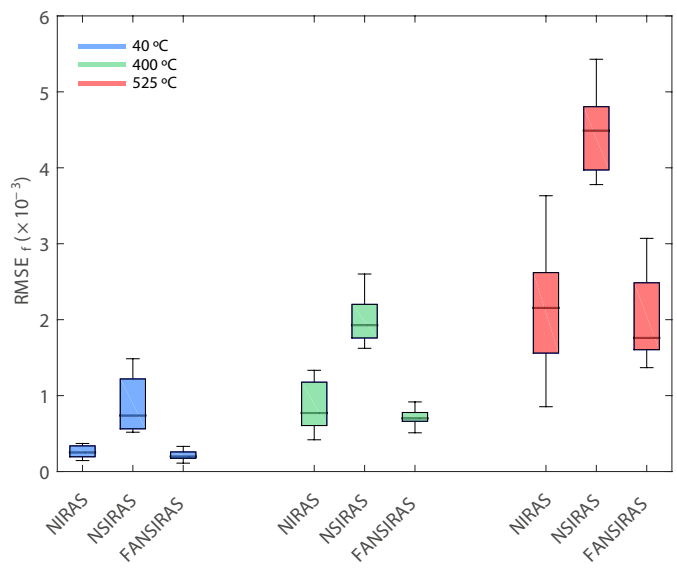

(a)

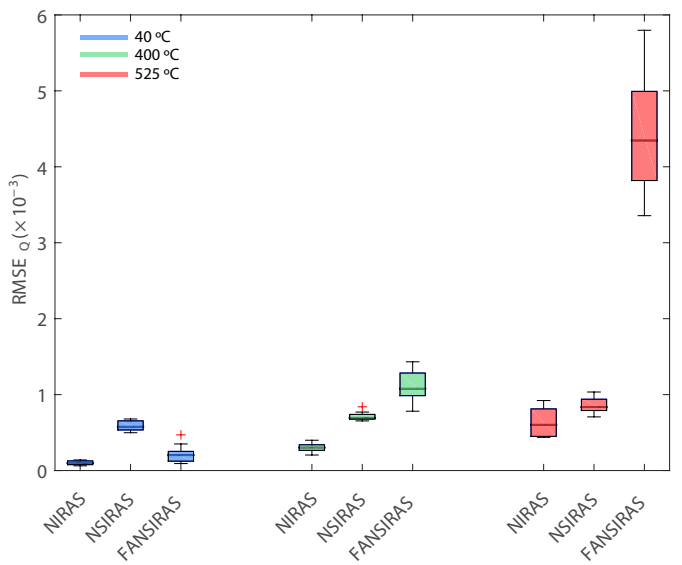

(b)

Figure 17: Comparing boxplots of the root mean square error (RMSE) for the amplitude-dependent dynamic features at different temperatures $40^{\circ} \mathrm{C}$ (blue),

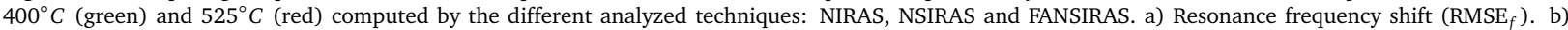
Attenuation $\left(\mathrm{RMSE}_{Q}\right)$.

\section{Conclusions}

In this study, a new method to evaluate non-linear behaviour in materials is presented. NIRAS is the traditional resonant method to determine the non-linearity of mesoscopic materials; it uses several impacts. The proposed socalled FANSIRAS procedure is focused on obtaining the same results as the traditional technique but by analysing only a single impact. Both techniques, along with the NSIRAS procedure, have been analyzed in detail, based on their mathematical backgrounds, and compared in terms of their estimations for a proposed simulated model and the damage level of heat-exposed mortar.

The FANSIRAS algorithm takes advantage of the appropriate processing of a single reverberation signal to 
estimate equivalent acquisitions to those obtained from NIRAS with its several blow impacts. This brand new

455 procedure respects the underlying phenomena, composed of both an exponentially decaying signal and a timevarying phase signal, which results in the feasibility of using a single impact to evaluate the non-linear features of damaged materials. Both, the theoretical and the experimental results have shown that the downward frequency shifts and the damping changes are equivalent for NIRAS and FANSIRAS techniques. It has been concluded that the variation of the damping factor $Q$ with the amplitude is exclusively sensitive to changes on the bandwidth. There is not an amplitude-dependent attenuation. Moreover, a special effort has been made to standardize the technique regardless of the acquisition equipment (accelerometer sensitivity, $f_{s}, N$ ) and the computation variables $\left(N_{D F T}\right)$ in order for the results to be reproducible in further studies. The application of the FANSIRAS analysis may have important uses in industrial applications in that the quality control can then be conducted through a single resonance frequency measurement.

\section{Acknowledgements}

This work has been supported by the Spanish Administration under grants TEC2011-23403, BES2015-071469, BIA2014-55311-C2-1-P and BIA2014-55311-C2-2-P. The authors are also grateful for FEDER funds.

\section{References}

[1] R. A. Guyer, P. A. Johnson, Nonlinear Mesoscopic Elasticity: Evidence for a New Class of Materials, Physics Today 52 (4) (1999) 30.

[2] K. R. McCall, R. A. Guyer, Equation of state and wave propagation in hysteretic nonlinear elastic materials, Journal of Geophysical Research: Solid Earth 99 (B12) (1994) 23887-23897, ISSN 2156-2202.

[3] M. Bentahar, H. El Aqra, R. El Guerjouma, M. Griffa, M. Scalerandi, Hysteretic elasticity in damaged concrete: Quantitative analysis of slow and fast dynamics, Phys. Rev. B 73 (2006) 014116.

[4] P. A. Johnson, B. Zinszner, P. N. Rasolofosaon, Resonance and elastic nonlinear phenomena in rock, Journal of Geophysical Research 101 (1996) 11-553.

[5] P. Delsanto, M. Scalerandi, Modeling nonclassical nonlinearity, conditioning, and slow dynamics effects in mesoscopic elastic materials, Physical Review B 68 (6) (2003) 1-9, ISSN 0163-1829.

[6] K. Van Den Abeele, J. Carmeliet, J. A. Ten Cate, P. Johnson, Nonlinear ElasticWave Spectroscopy (NEWS) Techniques to Discern Material Damage, Part I: Nonlinear Wave Modulation Spectroscopy (NWMS), Research in Nondestructive Evaluation 12 (1) (2000) 17-30, ISSN 0934-9847.

[7] K. Van Den Abeele, W. Desadeleer, G. De Schutter, M. Wevers, Active and passive monitoring of the early hydration process in concrete using linear and nonlinear acoustics, Cement and concrete research 39 (5) (2009) 426-432, ISSN 0008-8846.

[8] K. Van Den Abeele, P. A. Johnson, A. Sutin, Nonlinear Elastic Wave Spectroscopy (NEWS) Techniques to Discern Material Damage, Part II: Single-Mode Nonlinear Resonance Acoustic Spectroscopy, Research in Nondestructive Evaluation 12 (1) (2000) 17-30, ISSN 0934-9847.

[9] D. Broda, W. Staszewski, A. Martowicz, T. Uhl, V. Silberschmidt, Modelling of nonlinear crack wave interactions for damage detection based on ultrasound. A review, Journal of Sound and Vibration 333 (4) (2014) 1097 - 1118, ISSN 0022-460X.

[10] J. Jin, M. G. Moreno, J. Riviere, P. Shokouhi, Impact-Based Nonlinear Acoustic Testing for Characterizing Distributed Damage in Concrete, Journal of Nondestructive Evaluation 36 (3) (2017) 51, ISSN 1573-4862.

[11] K. Van Den Abeele, P. Y. Le Bas, B. Van Damme, T. Katkowski, Quantification of material nonlinearity in relation to microdamage density using nonlinear reverberation spectroscopy: Experimental and theoretical study., The Journal of the Acoustical Society of America 126 (3) (2009) 963-72, ISSN 1520-8524.

[12] C. Payan, V. Garnier, J. Moysan, P. A. Johnson, Applying nonlinear resonant ultrasound spectroscopy to improving thermal damage assessment in concrete, The Journal of the Acoustical Society of America 121 (4) (2007) EL125, ISSN 00014966.

[13] C. Payan, T. J. Ulrich, P. Y. Le Bas, T. Saleh, M. Guimaraes, Quantitative linear and nonlinear resonance inspection techniques and analysis for material characterization: Application to concrete thermal damage., The Journal of the Acoustical Society of America 136 (2) (2014) 537, ISSN 1520-8524.

[14] G. J. Kim, S. J. Park, H. G. Kwak, Experimental characterization of ultrasonic nonlinearity in concrete under cyclic change of prestressing force using Nonlinear Resonant Ultrasonic Spectroscopy, Construction and Building Materials 157 (2017) $700-707$.

[15] S. J. Park, G. J. Kim, H. G. Kwak, Characterization of stress-dependent ultrasonic nonlinearity variation in concrete under cyclic loading using nonlinear resonant ultrasonic method, Construction and Building Materials 145 (2017) 272-282. 
[16] J. Chen, A. R. Jayapalan, J. Y. Kim, K. E. Kurtis, L. J. Jacobs, Rapid evaluation of alkali-silica reactivity of aggregates using a nonlinear resonance spectroscopy technique, Cement and Concrete Research 40 (6) (2010) 914-923, ISSN 00088846.

[17] K. J. Leśnicki, J. Y. Kim, K. E. Kurtis, L. J. Jacobs, Characterization of ASR damage in concrete using nonlinear impact resonance acoustic spectroscopy technique, NDT \& E International 44 (8) (2011) 721-727, ISSN 09638695.

505

[18] V. Genovés, L. Soriano, M. V. Borrachero, J. Eiras, J. Payá, Preliminary study on short-term sulphate attack evaluation by non-linear impact resonance acoustic spectroscopy technique, Construction and Building Materials 78 (2015) 295-302, ISSN 09500618.

[19] J. N. Eiras, J. Monzó, J. Payá, T. Kundu, J. S. Popovics, Non-classical nonlinear feature extraction from standard resonance vibration data for damage detection, The Journal of the Acoustical Society of America 135 (2) (2014) EL82-EL87, ISSN 0001-4966.

510 [20] U. Dahlen, N. Ryden, A. Jakobsson, Damage identification in concrete using impact non-linear reverberation spectroscopy, NDT \& E International 75 (2015) 15-25, ISSN 09638695.

[21] L. Cohen, Time-frequency analysis, vol. 778, Prentice hall, 1995.

[22] S. J. Park, H. J. Yim, H. G. Kwak, Nonlinear resonance vibration method to estimate the damage level on heat-exposed concrete, Fire Safety Journal 69 (2014) 36-42, ISSN 03797112

515 [23] M. Scalerandi, M. Bentahar, C. Mechri, Conditioning and elastic nonlinearity in concrete: Separation of damping and phase contributions, Construction and Building Materials 161 (2018) 208-220.

[24] J. A. TenCate, E. Smith, R. A. Guyer, Universal slow dynamics in granular solids, Physical Review Letters 85 (5) (2000) 1020. 